\title{
EXCLUDING KURATOWSKI GRAPHS AND THEIR DUALS FROM BINARY MATROIDS
}

\author{
DILLON MAYHEW, GORDON ROYLE, GEOFF WHITTLE
}

\begin{abstract}
We consider various applications of our characterization of the internally 4 -connected binary matroids with no $M\left(K_{3,3}\right)$-minor. In particular, we characterize the internally 4-connected members of those classes of binary matroids produced by excluding any collection of cycle and bond matroids of $K_{3,3}$ and $K_{5}$, as long as that collection contains either $M\left(K_{3,3}\right)$ or $M^{*}\left(K_{3,3}\right)$. We also present polynomial-time algorithms for deciding membership of these classes, where the input consists of a matrix with entries from $\mathrm{GF}(2)$. In addition we characterize the maximum-sized simple binary matroids with no $M\left(K_{3,3}\right)$-minor, for any particular rank, and we show that a binary matroid with no $M\left(K_{3,3}\right)$-minor has a critical exponent over $\mathrm{GF}(2)$ of at most four.
\end{abstract}

\section{INTRODUCTION}

In a previous article we proved the following theorem.

Theorem 1.1. [12, Theorem 1.1] An internally 4-connected binary matroid $M$ has no minor isomorphic to $M\left(K_{3,3}\right)$ if and only if $M$ is either:

(i) cographic;

(ii) isomorphic to a triangular or triadic Möbius matroid; or,

(iii) isomorphic to one of 18 sporadic matroids.

The Möbius matroids are single-element extensions of bond matroids of Möbius ladders. We will describe them in Section 2.5. The 18 sporadic matroids appearing in Theorem 1.1 have ground sets of cardinality at most 21 , and have rank at most 11. Matrix representations of the sporadic matroids appear in Appendix B of [12], and are also available from the website of the second author (http://www. maths.uwa.edu.au/ gordon).

This sequel explores various applications of Theorem 1.1. In Sections 1.1 to 1.4 we introduce these applications and state our main results. In Section 1.5 we collect some conjectures motivated by the results in this paper. Before proceeding, we note that the proof of Theorem 1.1 involves a considerable amount of computer checking. In this paper we also use a computer to check a handful of statements. For this purpose we use MACEK, the software package developed by Petr Hliněný. MACEK is freely available to download, along with supporting documentation, at the current website http://www.fi.muni.cz/ hlineny/MACEK. 
1.1. Other classes. In Section 3 we characterize the internally 4-connected binary matroids with no minors in $\mathcal{M}$, where $\mathcal{M}$ is some subset of $\left\{M\left(K_{3,3}\right), M\left(K_{5}\right), M^{*}\left(K_{3,3}\right), M^{*}\left(K_{5}\right)\right\}$ such that $\mathcal{M}$ contains either $M\left(K_{3,3}\right)$ or $M^{*}\left(K_{3,3}\right)$. Thus we characterize the internally 4-connected members in twelve different families of binary matroids. Only the smallest of these classes has been characterized before [16].

1.2. Polynomial-time algorithms. In Section 4 we consider algorithmic consequences of Theorem 1.1. We present a polynomial-time algorithm for deciding if a binary matroid (represented by a matrix over $\mathrm{GF}(2)$ ) has a minor in $\mathcal{M}$, where $\mathcal{M}$ is a subset of $\left\{M\left(K_{3,3}\right), M\left(K_{5}\right), M^{*}\left(K_{3,3}\right), M^{*}\left(K_{5}\right)\right\}$, and $\mathcal{M}$ contains either $M\left(K_{3,3}\right)$ or $M^{*}\left(K_{3,3}\right)$. We also consider oracle algorithms.

Algorithms for binary matroids. Seymour's [18] famous decomposition theorem for regular matroids leads to a polynomial-time algorithm for deciding whether a matrix over $\mathrm{GF}(2)$ represents a regular matroid. In Section 4 we develop analogous algorithms for recognizing the twelve classes of binary matroids described in Section 3. The main theorem of Section 4 is the following result.

Theorem 1.2. Suppose that $\mathcal{M}$ is a subset of $\left\{M\left(K_{3,3}\right), M\left(K_{5}\right)\right.$, $\left.M^{*}\left(K_{3,3}\right), M^{*}\left(K_{5}\right)\right\}$ such that $\mathcal{M}$ contains either $M\left(K_{3,3}\right)$ or $M^{*}\left(K_{3,3}\right)$. There is a polynomial-time algorithm for solving the following problem: Given a matrix $A$ over $\mathrm{GF}(2)$, decide whether $M[A]$ has a minor in $\mathcal{M}$.

The algorithms of Theorem 1.2 are necessarily more complicated than the algorithm for recognizing regular matroids, for the class of regular matroids is closed under 3-sums, while the classes in Theorem 1.2 are not. Much of Section 4.1 is spent developing more sophisticated ways of decomposing a binary matroid into its internally 4 -connected components.

Oracle algorithms. Suppose that $M$ is a matroid on the ground set $E$. When queried about a subset $X \subseteq E$ a matroid oracle returns in unit time some information about $X$. That information is typically the rank of $X$, or an answer to the question 'Is $X$ independent?'. An oracle algorithm is efficient if the number of calls it makes to the oracle is bounded by some fixed polynomial function of $|E(M)|$, for any matroid $M$, and all additional computation can also be done in polynomial time.

Using Seymour's decomposition theorem, and techniques invented by Truemper [20], it is possible to construct an efficient oracle algorithm for deciding whether a matroid is regular (see [1, Section 7.4] and [21]). In contrast to this, the algorithms of Theorem 1.2 do not extend to efficient oracle algorithms, as we now discuss. An example of Seymour's [19] shows that there is no efficient oracle algorithm for deciding whether a matroid is binary. This same example shows that if $\mathcal{M}$ is any collection of bond 
and cycle matroids of Kuratowski graphs, then there can be no efficient oracle algorithm that decides if a matroid is binary with no minor in $\mathcal{M}$ (see Proposition 4.20). Thus we can expect no oracle analogue of Theorem 1.2.

On the other hand, the characterizations of Section 3 feature basic classes that are recognizable by efficient oracle algorithms. This reveals the curious fact that it is possible to have an efficient algorithm for deciding membership in a class of matroids when the input is guaranteed to be internally 4-connected, even if there is no efficient algorithm for deciding membership in the general case (see Propositions 4.20 and 4.21). We summarize this phenomenon in the following corollary.

Corollary 1.3. Let $\mathcal{M}$ be a subset of $\left\{M\left(K_{3,3}\right), M\left(K_{5}\right), M^{*}\left(K_{3,3}\right)\right.$, $\left.M^{*}\left(K_{5}\right)\right\}$ such that $\mathcal{M}$ contains either $M\left(K_{3,3}\right)$ or $M^{*}\left(K_{3,3}\right)$. There is no efficient oracle algorithm for deciding whether a matroid belongs to the class of binary matroids with no minor in $\mathcal{M}$. However, there is an efficient oracle algorithm for deciding whether an internally 4-connected matroid is a binary matroid with no minor in $\mathcal{M}$.

Oracle algorithms are discussed more fully in Section 4.2.

1.3. Maximum-sized binary matroids with no $M\left(K_{3,3}\right)$. In Section 5 we use Theorem 1.1 to determine the maximum size of a simple rank- $r$ binary matroid with no $M\left(K_{3,3}\right)$-minor. Moreover, we characterize the matroids that obtain this upper bound. This completely resolves a question studied by Kung [10]. He showed that a simple rank- $r$ binary matroid $M$ without an $M\left(K_{3,3}\right)$-minor has at most $10 r$ elements. Theorem 5.3 shows that, in fact, $|E(M)| \leq 14 r / 3-\alpha(r)$, where $\alpha(r)$ assumes one of three values depending on the residue of $r$ modulo 3. Moreover this bound is sharp. Any matroid meeting this bound can be obtained by starting with either $\operatorname{PG}(1,2)$, $\mathrm{PG}(2,2)$, or $\mathrm{PG}(3,2)$, and then repeatedly adding copies of $\mathrm{PG}(3,2)$ via parallel connections.

1.4. Critical exponents. If $M$ is a matroid then its characteristic polynomial, $\chi(M ; t)$ is a polynomial in the variable $t$. If $M$ is loopless and representable over $\operatorname{GF}(q)$, then the "critical exponent" of $M$ over $q$, denoted $c(M ; q)$, is the smallest positive integer $k$ such that $\chi\left(M ; q^{k}\right) \neq 0$. In Section 6 we show that any loopless binary matroid with no $M\left(K_{3,3}\right)$-minor has a critical exponent over $\mathrm{GF}(2)$ of at most four. Moreover, we characterize such matroids that have critical exponent equal to four: They are precisely those with a 3 -connected component isomorphic to $\mathrm{PG}(3,2)$ (see Theorem 6.2). This resolves a programme initiated by Kung [10], who showed that if $M$ is a simple binary matroid with no $M\left(K_{3,3}\right)$-minor, then $c(M ; 2) \leq 10$.

1.5. Conjectures on recognition problems. In this section we speculate on the extent to which the algorithmic results of Section 4 are special cases of more general theorems. While this paper focuses on binary matroids, the 
conjectures are also interesting for other fields and we state some of them at that level of generality. The first two conjectures are originally due to Neil Robertson and Paul Seymour, although apparently they never stated them in print. They are discussed in [5] in the context of extending the Graph Minors project of Robertson and Seymour to matroids representable over finite fields.

Conjecture 1.4 (Well-Quasi-Ordering Conjecture). Let $\mathrm{GF}(q)$ be a finite field. Then any infinite set of $\operatorname{GF}(q)$-representable matroids contains two matroids, one of which is isomorphic to a minor of the other.

A positive answer to Conjecture 1.4 would imply that any minor-closed class of $\operatorname{GF}(q)$-representable matroids has a finite number of $\mathrm{GF}(q)$-representable excluded minors.

Conjecture 1.5. For any finite field $\mathrm{GF}(q)$ and any $\mathrm{GF}(q)$-representable matroid $N$, there is a polynomial-time algorithm for solving the following problem: Given a matrix $A$ over $\operatorname{GF}(q)$, decide whether $M[A]$ has a minor isomorphic to $N$.

We note that a positive answer to Conjecture 1.5 implies the famous result by Robertson and Seymour [17] that there is polynomial-time algorithm for detecting the presence of a fixed minor in a graph.

If Conjectures 1.4 and 1.5 were true, then the next conjecture would also hold.

Conjecture 1.6. For any finite field $\operatorname{GF}(q)$, and any minor-closed class $\mathcal{M}$ of $\operatorname{GF}(q)$-representable matroids, there is a polynomial-time algorithm for solving the following problem: Given a matrix $A$ over $\mathrm{GF}(q)$, decide whether $M[A]$ belongs to $\mathcal{M}$.

The results of Section 4 show that Conjecture 1.6 holds for the classes of binary matroids described in Section 3.

Just as intriguing is the possibility of extending the oracle-complexity results mentioned in Corollary 1.3. In what follows we restrict our attention to binary matroids. Seymour's [19] example shows that there is no efficient oracle algorithm for deciding whether a matroid is binary. Because of this example, one might expect that finding efficient oracle algorithms for recognizing classes of binary matroids is a hopeless task. But Corollary 1.3 makes it plausible that the difficulties may simply be due to degeneracies caused by low connectivity.

Conjecture 1.7. There is an efficient oracle algorithm for deciding if an internally 4-connected matroid is binary.

This is an ambitious conjecture. The next conjecture is somewhat more modest.

Conjecture 1.8. Let $\mathcal{M}$ be a proper minor-closed class of binary matroids. There is an efficient oracle algorithm for deciding whether an internally 4-connected matroid belongs to $\mathcal{M}$. 
The matroids used by Seymour to construct his example are examples of "spikes". Spikes are a notorious source of difficulty in matroid theory. More generally, a spike-like flower of order $n$ in a 3 -connected matroid $M$ is a partition $\left(P_{1}, \ldots, P_{n}\right)$ of the ground set of $M$ such that, for every proper subset $J$ of $\{1, \ldots, n\}$ the partition

$$
\left(\bigcup_{j \in J} P_{j}, E(M)-\bigcup_{j \in J} P_{j}\right)
$$

is an exact 3 -separation of $M$; and, for all distinct $i$ and $j$ in $\{1, \ldots, n\}$ we have $r\left(P_{i} \cup P_{j}\right)=r\left(P_{i}\right)+r\left(P_{j}\right)-1$. A rank- $n$ spike contains a spike-like flower of order $n$. We believe the existence of large spike-like flowers is at the heart of the difficulty of recognising binary matroids. This belief is encapsulated by the next conjecture, which is a strengthening of Conjecture 1.7.

Conjecture 1.9. Let $k$ be a fixed positive integer. There is an efficient oracle algorithm for deciding if a 3-connected matroid with no spike-like flower of order $k$ is a binary matroid.

Indeed the hypothesis of 3-connectivity in Conjecture 1.9 could be removed modulo the annoying technicalities of stating what it means for a more general matroid to have a spike-like flower. In fact, it is probably not difficult to prove that Conjecture 1.9 follows from Conjecture 1.7. Similar comments could be made about an analogous generalization of Conjecture 1.8

\section{Preliminaries}

Our reference for fundamental notions of matroid theory is Oxley [15], and our notation follows that source, except that we denote the simple matroid associated with the matroid $M$ by $\operatorname{si}(M)$. We assume that the ground set of $\operatorname{si}(M)$ is the set of parallel classes of $M$. If $\mathcal{M}$ is a collection of binary matroids, then $\mathcal{E} \mathcal{X}(\mathcal{M})$ is the family of binary matroids with no minors in $\mathcal{M}$.

2.1. Connectivity. Let $M$ be a matroid on the ground set $E$. The connectivity function of $M$, denoted by $\lambda_{M}$, takes any subset $X \subseteq E$ to $r_{M}(X)+r_{M}(E-X)-r(M)$. We use $\lambda_{M}^{*}$ to denote the connectivity function of $M^{*}$. A partition $\left(X_{1}, X_{2}\right)$ of $E$ is a $k$-separation of $M$ if $\left|X_{1}\right|,\left|X_{2}\right| \geq k$ and $\lambda_{M}\left(X_{1}\right)=\lambda_{M}\left(X_{2}\right)<k$. A $k$-separation $\left(X_{1}, X_{2}\right)$ is exact if $\lambda_{M}\left(X_{1}\right)=k-1$. We say that $M$ is $n$-connected if it has no $k$-separations such that $k<n$. In addition $M$ is internally 4-connected if it is 3 -connected and whenever $\left(X_{1}, X_{2}\right)$ is a 3 -separation, then $\min \left\{\left|X_{1}\right|,\left|X_{2}\right|\right\}=3$.

The next result follows directly from a theorem of Oxley [14, Theorem 3.6].

Lemma 2.1. Let $T$ be a triangle of a 3-connected binary matroid $M$. If the rank and corank of $M$ are at least three then $M$ has an $M\left(K_{4}\right)$-minor in which $T$ is a triangle. 
2.2. Symmetric difference of matroids. Suppose that $M$ is a binary matroid. A cycle of $M$ is a subset $Z$ of $E(M)$ such that $Z$ can be expressed as a (possibly empty) disjoint union of circuits of $M$. The symmetric difference of sets $Z_{1}$ and $Z_{2}$ is denoted by $Z_{1} \triangle Z_{2}$. Binary matroids are characterized by the fact that the symmetric difference of any two cycles is another cycle.

Let $M_{1}$ and $M_{2}$ be two binary matroids on the ground sets $E_{1}$ and $E_{2}$ respectively. Let $\mathcal{Z}$ be the collection

$$
\left\{Z_{1} \triangle Z_{2} \subseteq E_{1} \triangle E_{2} \mid Z_{i} \text { is a cycle of } M_{i}, i=1,2\right\} .
$$

Then $\mathcal{Z}$ is the collection of cycles of a binary matroid on the ground set $E_{1} \triangle E_{2}$. We denote this matroid $M_{1} \triangle M_{2}$.

Proposition 2.2. $[18,(4.4)]$ Suppose that $M_{1}$ and $M_{2}$ are binary matroids on the sets $E_{1}$ and $E_{2}$ respectively. If $A$ and $B$ are disjoint subsets of $E_{1}-E_{2}$ then

$$
\left(M_{1} \triangle M_{2}\right) / A \backslash B=\left(M_{1} / A \backslash B\right) \triangle M_{2} .
$$

The next two results are straightforward to prove.

Proposition 2.3. Suppose that $M_{1}$ and $M_{2}$ are binary matroids on the ground sets $E_{1}$ and $E_{2}$ respectively. Let $T=E_{1} \cap E_{2}$, and assume that $M_{1}\left|T=M_{2}\right| T$. Then

$$
\left(M_{1} \triangle M_{2}\right)\left|\left(E_{1}-T\right)=M_{1}\right|\left(E_{1}-T\right) .
$$

Proposition 2.4. Suppose that $M_{1}, M_{2}$, and $M_{3}$ are binary matroids on the ground sets $E_{1}, E_{2}$, and $E_{3}$ respectively. Then

$$
\left(M_{1} \triangle M_{2}\right) \triangle M_{3}=M_{1} \triangle\left(M_{2} \triangle M_{3}\right) .
$$

Proposition 2.5. Suppose that $M_{1}$ and $M_{2}$ are binary matroids on the ground sets $E_{1}$ and $E_{2}$ respectively. Assume $E_{1} \subseteq E_{2}$. If $r\left(M_{1}\right)=0$ then $M_{1} \triangle M_{2}=M_{2} / E_{1}$.

Proof. Suppose that $C$ is a circuit of $M_{2} / E_{1}$. Then $C \cup E_{1}$ contains a circuit $C^{\prime}$ of $M_{2}$ such that $C=C^{\prime}-E_{1}$. Now $C^{\prime} \cap E_{1}$ is a cycle of $M_{1}$ since it is a union of loops, so $C^{\prime}-E_{1}=C$ is a cycle of $M_{1} \triangle M_{2}$, and hence contains a circuit of $M_{1} \triangle M_{2}$. On the other hand, suppose that $C$ is a circuit of $M_{1} \triangle M_{2}$. Then $C=Z_{1} \triangle Z_{2}$, where $Z_{1}$ is a cycle of $M_{1}$ and $Z_{2}$ is a cycle of $M_{2}$. There must be some circuit $C^{\prime} \subseteq Z_{2}$ of $M_{2}$ such that $C^{\prime} \cap\left(E_{2}-E_{1}\right) \neq \varnothing$. Then $C^{\prime}$ contains a circuit of $M_{2} / E_{1}$. Thus every circuit of $M_{1} \triangle M_{2}$ contains a circuit of $M_{2} / E_{1}$ and we are done.

2.3. The $\Delta-Y$ operation. Suppose that $M$ is a binary matroid and assume that $T=\left\{a_{1}, a_{2}, a_{3}\right\}$ is a triangle of $M$. Let $N$ be an isomorphic copy of $M\left(K_{4}\right)$ such that $E(N) \cap E(M)=T$, where $T$ is a triangle of $N$. Assume that $E(N)=T \cup\left\{a_{1}^{\prime}, a_{2}^{\prime}, a_{3}^{\prime}\right\}$, where $\left(\left\{a_{1}^{\prime}, a_{2}^{\prime}, a_{3}^{\prime}\right\}-a_{i}^{\prime}\right) \cup a_{i}$ is a triangle of $N$ for $i=1,2,3$. We say that $N \triangle M$ is produced from $M$ by a $\Delta-Y$ operation on $T$, and we use $\Delta_{T}(M)$ to denote the resulting matroid. To 
ensure that $M$ and $\Delta_{T}(M)$ have the same ground set we relabel $a_{i}^{\prime}$ with $a_{i}$ in $\Delta_{T}(M)$, for $i=1,2,3$.

Oxley, Semple, and Vertigan [13] generalize the $\Delta-Y$ operation using Brylawski's parallel connection [2]. It is easy to see that if $T$ is coindependent in $M$, then the two definitions coincide. Many of the following results are identical to those in [13]. Because our definition of the $\Delta-Y$ operation is slightly different we provide some independent proofs.

Proposition 2.6. Suppose that $T$ is a coindependent triangle of the binary matroid $M$. Then $T$ is a triad of $\Delta_{T}(M)$.

Proof. Suppose $\Delta_{T}(M)=N \triangle M$. Recall that the elements of $E(N)-T$ are relabeled in $\Delta_{T}(M)$, so that $\Delta_{T}(M)$ and $M$ have the same ground set. Now $E(N)-T$ is a cocircuit of $N$, and hence a cocircuit of $N / T$. Because $T$ is coindependent in $M$ it follows that $T$ is a set of loops in $M / E(M)-T$. Propositions 2.2 and 2.5 imply that

$$
N / T=N \triangle(M / E(M)-T)=(N \triangle M) / E(M)-T .
$$

Therefore $T$ is a cocircuit of $N \triangle M=\Delta_{T}(M)$.

The next results follow easily from Propositions 2.3 and 2.4.

Proposition 2.7. Suppose that $T$ is a triangle of the binary matroid $M$. Then $\Delta_{T}(M) \backslash T=M \backslash T$.

Proposition 2.8. Suppose that $M$ is a binary matroid and that $T_{1}$ and $T_{2}$ are disjoint triangles of $M$. Then

$$
\Delta_{T_{1}}\left(\Delta_{T_{2}}(M)\right)=\Delta_{T_{2}}\left(\Delta_{T_{1}}(M)\right) .
$$

Proposition 2.9. Suppose that $T$ is a triangle of the binary matroid $M$ and that $a \in T$. Then $\Delta_{T}(M) / a=M \backslash a$.

Proof. Suppose that $\Delta_{T}(M)=N \triangle M$. Let $a^{\prime}$ be the element of $E(N)-T$ that is relabeled with $a$. Proposition 2.2 says that $(N \triangle M) / a^{\prime}=\left(N / a^{\prime}\right) \triangle M$. Note that $N / a^{\prime}$ consists of the triangle $T$ with parallel elements added to both members of $T-a$. Suppose that $\left\{b, b^{\prime}\right\}$ is a parallel pair of $N / a^{\prime}$, where $b \in T$. Let $M^{+}$be obtained from $M$ by adding $x$ in parallel to $b$. Then $\{b, x\}$ is a cycle of $M^{+}$, so $\left\{b^{\prime}, x\right\}$ is a cycle of $\left(N / a^{\prime}\right) \triangle M^{+}$. Hence either $\left\{b^{\prime}, x\right\}$ is a parallel pair, or both $b^{\prime}$ and $x$ are loops in $\left(N / a^{\prime}\right) \triangle M^{+}$. In either case Proposition 2.2 implies that

$$
\begin{aligned}
\left(N / a^{\prime}\right) \triangle M=\left(N / a^{\prime}\right) \triangle\left(M^{+} \backslash x\right)= & \left(\left(N / a^{\prime}\right) \triangle M^{+}\right) \backslash x \cong \\
& \left(\left(N / a^{\prime}\right) \triangle M^{+}\right) \backslash b^{\prime}=\left(N / a^{\prime} \backslash b^{\prime}\right) \triangle M^{+} .
\end{aligned}
$$

By using the same argument again we can show that $\left(N / a^{\prime}\right) \triangle M$ is isomorphic to the symmetric difference of $N \mid T$ and the matroid obtained from $M$ by adding parallel elements to the members of $T-a$. Now the result follows easily from Proposition 2.3. 
Proposition 2.10. Suppose that $T$ is a triangle of the binary matroid $M$. Then $r\left(\Delta_{T}(M)\right)=r(M)+1$.

Proof. Let $a$ be an element of $T$. Since $a$ is not a coloop in $M$, nor a loop in $\Delta_{T}(M)$ the result follows immediately from Proposition 2.9.

2.4. Matroid sums. In this section we define matroid 1-, 2-, and 3-sums, following the route taken by Seymour [18]. Suppose that $M_{1}$ and $M_{2}$ are binary matroids on the ground sets $E_{1}$ and $E_{2}$ respectively. If $E_{1}$ and $E_{2}$ are disjoint, and neither $E_{1}$ nor $E_{2}$ is empty, then $M_{1} \triangle M_{2}$ is the 1-sum of $M_{1}$ and $M_{2}$, denoted $M_{1} \oplus M_{2}$. If $E_{1}$ and $E_{2}$ meet in a single element $p$, where $p$ is a loop or coloop in neither $M_{1}$ nor $M_{2}$, and $\left|E_{1}\right|,\left|E_{2}\right| \geq 3$, then $M_{1} \triangle M_{2}$ is the 2-sum of $M_{1}$ and $M_{2}$, denoted $M_{1} \oplus_{2} M_{2}$. We say that $p$ is the basepoint of the 2-sum. Finally, suppose that $E_{1} \cap E_{2}=T$ and assume that the following conditions hold:

(i) $T$ is a triangle in both $M_{1}$ and $M_{2}$;

(ii) $T$ contains a cocircuit in neither $M_{1}$ nor $M_{2}$; and,

(iii) $\left|E_{1}\right|,\left|E_{2}\right| \geq 7$.

In this case $M_{1} \triangle M_{2}$ is the 3 -sum of $M_{1}$ and $M_{2}$, denoted $M_{1} \oplus_{3} M_{2}$.

Next we list a number of results due to Seymour.

Proposition 2.11. [18, (2.6)] Suppose that $\left(X_{1}, X_{2}\right)$ is an exact 2-separation of the binary matroid $M$. Then there are binary matroids $M_{1}$ and $M_{2}$ on the ground sets $X_{1} \cup p$ and $X_{2} \cup p$, where $p \notin X_{1} \cup X_{2}$, such that $M=M_{1} \oplus_{2}$ $M_{2}$. Conversely, if $M=M_{1} \oplus_{2} M_{2}$ then $\left(E\left(M_{1}\right)-E\left(M_{2}\right), E\left(M_{2}\right)-E\left(M_{1}\right)\right)$ is a 2-separation of $M$, and $M_{1}$ and $M_{2}$ are isomorphic to minors of $M$.

Proposition 2.12. [18, (2.9)] Suppose that $\left(X_{1}, X_{2}\right)$ is an exact 3-separation of the binary matroid $M$ such that $\min \left\{\left|X_{1}\right|,\left|X_{2}\right|\right\} \geq 4$. Then there are binary matroids $M_{1}$ and $M_{2}$ on the ground sets $X_{1} \cup T$ and $X_{2} \cup T$ respectively, where $T$ is disjoint from $X_{1} \cup X_{2}$, such that $M=M_{1} \oplus_{3} M_{2}$. Conversely, if $M=M_{1} \oplus_{3} M_{2}$, then $\left(E\left(M_{1}\right)-E\left(M_{2}\right), E\left(M_{2}\right)-E\left(M_{1}\right)\right)$ is an exact 3-separation of $M$.

Proposition 2.13. [18, (4.1)] Suppose that the binary matroid $M$ can be expressed as the 3-sum of $M_{1}$ and $M_{2}$. If $M$ is 3-connected then $M_{1}$ and $M_{2}$ are isomorphic to minors of $M$.

Proposition 2.14. $[18,(4.3)]$ Suppose that $M_{1}$ and $M_{2}$ are binary matroids on the ground sets $E_{1}$ and $E_{2}$ respectively. Suppose also that the 3 -sum of $M_{1}$ and $M_{2}$ is defined and that $M_{1} \oplus_{3} M_{2}$ is 3-connected. If $\left(X_{1}, X_{2}\right)$ is a 2-separation of $M_{1}$, then for some $i \in\{1,2\}$ we have that $X_{i}=\{x, z\}$, where $x \in E_{1}-E_{2}$ and $z \in E_{1} \cap E_{2}$. Moreover $x$ and $z$ are parallel in $M_{1}$.

Proposition 2.15. Suppose that $M_{1}$ and $M_{2}$ are binary matroids on the ground sets $E_{1}$ and $E_{2}$ respectively, where $E_{1} \cap E_{2}=T$. Suppose also that the 3-sum of $M_{1}$ and $M_{2}$ is defined. Let $M=M_{1} \oplus_{3} M_{2}$. If $M$ is 3-connected then $\Delta_{T}\left(M_{1}\right)$ and $\Delta_{T}\left(M_{2}\right)$ are isomorphic to minors of $M$. 
Proof. Suppose that $r\left(M_{1}\right)<3$. Proposition 2.14 implies that $M_{1}$ can have no parallel class containing more than two elements. Thus $\left|E_{1}\right| \leq 6$, a contradiction, as $M_{1} \oplus_{3} M_{2}$ is defined. Therefore $r\left(M_{1}\right) \geq 3$.

Suppose that $r\left(E_{1}-\mathrm{cl}_{M_{1}}(T)\right)<r\left(M_{1}\right)$. Since $r\left(M_{1}\right) \geq 3$, it follows that $E_{1}-\mathrm{cl}_{M_{1}}(T)$ is non-empty. If $\left|E_{1}-\mathrm{cl}_{M_{1}}(T)\right|=1$ then $M_{1}$ contains a coloop, and it is easy to see that this implies that $M_{1} \oplus_{3} M_{2}$ has a coloop, a contradiction. Therefore $\left|E_{1}-\mathrm{cl}_{M_{1}}(T)\right| \geq 2$, and $\left(\mathrm{cl}_{M_{1}}(T), E_{1}-\mathrm{cl}_{M_{1}}(T)\right)$ is a 2-separation of $M_{1}$. This contradicts Proposition 2.14 .

Therefore there is a basis $B$ of $M_{1}$ that avoids $\mathrm{cl}_{M_{1}}(T)$. Proposition 2.14 implies $\mathrm{si}\left(M_{1}\right)$ is 3 -connected and that the only parallel pairs of $M_{1}$ are contained in $\operatorname{cl}_{M_{1}}(T)$. Recall that the ground set of $\operatorname{si}\left(M_{1}\right)$ is the set of parallel classes of $M_{1}$. Thus $\mathrm{cl}_{M_{1}}(T)$ is a triangle of $\operatorname{si}\left(M_{1}\right)$. Since $B$ is a basis of $\operatorname{si}\left(M_{1}\right)$ and $\operatorname{cl}_{M_{1}}(T)$ avoids $B$ it follows that the rank and corank of $\operatorname{si}\left(M_{1}\right)$ are both at least three. Now Lemma 2.1 implies that $\operatorname{si}\left(M_{1}\right)$ has a minor isomorphic to $M\left(K_{4}\right)$ in which $\operatorname{cl}_{M_{1}}(T)$ is a triangle. Let $M^{\prime}$ be a minor of $M_{1}$ such that $M^{\prime} \cong M\left(K_{4}\right)$ and $T$ is a triangle of $M^{\prime}$. Proposition 2.2 says that $M^{\prime} \triangle M_{2}$ is a minor of $M$. However $M^{\prime} \triangle M_{2}$ is isomorphic to $\Delta_{T}\left(M_{2}\right)$. The same argument shows that $\Delta_{T}\left(M_{1}\right)$ is isomorphic to a minor of $M$.

Proposition 2.16. Suppose that $M_{1}$ and $M_{2}$ are binary matroids on the ground sets $E_{1}$ and $E_{2}$, where $E_{1} \cap E_{2}=T$ and $M_{1} \oplus_{3} M_{2}$ is defined. Let $T_{0}$ be a triangle of $M_{1}$ that is disjoint from $T$. Then $\Delta_{T_{0}}\left(M_{1} \oplus_{3} M_{2}\right)=$ $\Delta_{T_{0}}\left(M_{1}\right) \oplus_{3} M_{2}$.

Proof. Note that $T_{0}$ is a triangle of $M_{1} \oplus_{3} M_{2}$ by Proposition 2.3. Thus $\Delta_{T_{0}}\left(M_{1} \oplus_{3} M_{2}\right)$ is defined. Moreover $T$ is a triangle of $\Delta_{T_{0}}\left(M_{1}\right)$ for the same reason. Let $\Delta_{T_{0}}\left(M_{1}\right)=N \triangle M_{1}$, where $N \cong M\left(K_{4}\right)$. If $T$ contains a cocircuit in $N \triangle M_{1}$ then it contains a cocircuit in $\left(N \triangle M_{1}\right) /\left(E(N)-T_{0}\right)=$ $\left(N /\left(E(N)-T_{0}\right)\right) \triangle M_{1}$, which is equal to $M_{1} / T_{0}$ by Proposition 2.5. Thus $T$ contains a cocircuit in $M_{1}$, a contradiction as $M_{1} \oplus_{3} M_{2}$ is defined. Finally we observe that $\left|E\left(\Delta_{T_{0}}\left(M_{1}\right)\right)\right| \geq 7$. Therefore $\Delta_{T_{0}}\left(M_{1}\right) \oplus_{3} M_{2}$ is defined and Proposition 2.4 says that it is equal to $\Delta_{T_{0}}\left(M_{1} \oplus_{3} M_{2}\right)$.

It is well known that if $M_{0}$ is a 2- (respectively, 3-) connected matroid, and neither $M_{1}$ nor $M_{2}$ has an $M_{0}$-minor, then the 1- (respectively, 2-) sum of $M_{1}$ and $M_{2}$ has no $M_{0}$-minor. We would like an analogue of this fact for 3-sums, but unfortunately the strict analogue is false: For example, the binary matroid $R_{12}$ used by Seymour in his decomposition theorem for regular matroids can be expressed as the 3 -sum of $M\left(K_{5} \backslash e\right)$ and $M^{*}\left(K_{3,3}\right)$. Neither of these matroids has an $M\left(K_{3,3}\right)$-minor, but $R_{12}$ does. However, Lemma 2.19 contains a partial results in this direction. Before we can prove the lemma, we need two preliminary propositions.

Proposition 2.17. Suppose that $M_{1}$ and $M_{2}$ are binary matroids on the ground sets $E_{1}$ and $E_{2}$ respectively. Suppose that $E_{1} \cap E_{2}=\{e, f, g\}$, where $\{e, f, g\}$ is a triangle in $M_{2}$, and $e$ is a loop while $\{f, g\}$ is a parallel pair 
in $M_{1}$. Let $B_{2}$ be a basis of $M_{2}$ such that $B_{2} \cap\{e, f, g\}=\{e\}$. Then $B_{2}-e$ is a basis of $\left(M_{1} \triangle M_{2}\right) \mid\left(E_{2}-E_{1}\right)$.

Proof. Let $T=\{e, f, g\}$. Suppose that $B_{2}-e$ contains a circuit $C$ of $M_{1} \triangle M_{2}$. In this case $C=Z_{1} \triangle Z_{2}$, where $Z_{1} \subseteq T$ is a cycle of $M_{1}$ and $Z_{2} \subseteq B_{2} \cup T$ is a cycle of $M_{2}$. Suppose that $Z_{2}$ is the disjoint union of the circuits $C_{1}, \ldots, C_{t}$ of $M_{2}$. No circuit of $M_{2}$ can be contained in $B_{2}$, so $t \leq 2$ as $\left|T-B_{2}\right|=2$. Suppose that $t=1$. It cannot be the case that $f, g \in C_{1}$, for then the symmetric difference of $C_{1}$ and $T$ would be a circuit contained in $B_{2}$. Assume that $f \in C_{1}$. Then $Z_{2} \cap T=Z_{1}$ contains $f$, but not $g$. This is impossible as $Z_{1}$ is a cycle, and $f$ and $g$ are parallel in $M_{1}$. This contradiction shows that $t=2$. Since neither $C_{1}$ nor $C_{2}$ can contain $\{f, g\}$ we will assume that $f \in C_{1}$ and $g \in C_{2}$. As neither $C_{1}$ nor $C_{2}$ is contained in $T$ it follows that both meet $B_{2}-e$.

Suppose that $e$ is in neither $C_{1}$ nor $C_{2}$. Then $\left(C_{1}-f\right) \cup\{e, g\}$ and $C_{2}$ are distinct circuits contained in $B_{2} \cup g$, a contradiction as $B_{2}$ is a basis of $M_{2}$. Thus we will assume that $e \in C_{1}$ (the argument when $e \in C_{2}$ is identical). This implies that $\left(C_{1}-\{e, f\}\right) \cup g$ is a circuit, so $C_{2}$ must be equal to $\left(C_{1}-\{e, f\}\right) \cup g$. But this is a contradiction, as $C_{1}$ and $C_{2}$ are disjoint.

We have shown that $B_{2}-e$ is independent in $M_{1} \triangle M_{2}$. Let $x$ be an element in $E_{2}-\left(B_{2} \cup T\right)$. Then $B_{2} \cup x$ contains a circuit $C$ in $M_{2}$. As $B_{2}$ meets $T$ in $e$, and $e$ is a loop in $M_{1}$ it is now easy to see that $C-e$ is a cycle in $M_{1} \triangle M_{2}$. Thus $\left(B_{2}-e\right) \cup x$ is dependent in $M_{1} \triangle M_{2}$. This implies that $B_{2}-e$ is a basis of $E_{2}-T$ in $M_{1} \triangle M_{2}$, so we are done.

Proposition 2.18. Suppose that $M_{1}$ and $M_{2}$ are binary matroids on the ground sets $E_{1}$ and $E_{2}$ respectively. Suppose that $E_{1} \cap E_{2}=\{e, f, g\}$, where $\{e, f, g\}$ is a coindependent triangle in $M_{2}$, and $e$ is a loop while $\{f, g\}$ is a parallel pair in $M_{1}$. Then the restriction of $M_{1} \triangle M_{2}$ to $E_{2}-E_{1}$ is equal to $M_{2} / e \backslash f \backslash g$.

Proof. Let $T=\{e, f, g\}$. Let $C$ be a circuit of $M_{2} / e \backslash f \backslash g$. There is a circuit $C^{\prime} \subseteq C \cup e$ of $M_{2}$ such that $C^{\prime}-e=C$. As $e$ is a loop of $M_{1}$ it follows that $C^{\prime}-e=C$ is a cycle of $M_{1} \triangle M_{2}$, and hence contains a circuit of $M_{1} \triangle M_{2}$.

On the other hand, suppose that $I$ is an independent set of $M_{2} / e \backslash f \backslash g$. Then $I \cup e$ is independent in $M_{2}$, and as $T$ is coindependent in $M_{2}$ there is a basis $B_{2}$ of $M_{2}$ such that $I \cup e \subseteq B_{2}$ and $f, g \notin B_{2}$. Proposition 2.17 shows that $B_{2}-e$, and hence $I$, is independent in $M_{1} \triangle M_{2}$.

Suppose that $X$ is some subset of $E_{2}-T$. The previous arguments show that if $X$ is dependent in $M_{2} / e \backslash f \backslash g$ then it is dependent in $M_{1} \triangle M_{2}$, and if it is independent in $M_{2} / e \backslash f \backslash g$, then it is independent in $M_{1} \triangle M_{2}$. This completes the proof.

Lemma 2.19. Let $M_{0}$ be an internally 4-connected binary matroid such that $\left|E\left(M_{0}\right)\right| \geq 4$. Suppose that $M_{1}$ and $M_{2}$ are binary matroids on the ground sets $E_{1}$ and $E_{2}$ respectively and that neither $M_{1}$ nor $M_{2}$ has an $M_{0}$-minor. 
Assume that $E_{1} \cap E_{2}=T$ where $T$ is a triangle of both $M_{1}$ and $M_{2}$ and that the 3-sum of $M_{1}$ and $M_{2}$ is defined. If $M_{1} \oplus_{3} M_{2}$ has an $M_{0}$-minor then either $\Delta_{T}\left(M_{1}\right)$ or $\Delta_{T}\left(M_{2}\right)$ has an $M_{0}$-minor and $M_{0}$ contains at least one triad.

Proof. The hypotheses of the lemma imply that $T$ contains a cocircuit in neither $M_{1}$ nor $M_{2}$, and that $\left|E_{1}\right|,\left|E_{2}\right| \geq 7$. Let $M=M_{1} \oplus_{3} M_{2}$, so that $M=M_{1} \triangle M_{2}$. Proposition 2.12 asserts that $\left(E_{1}-T, E_{2}-T\right)$ is an exact 3 -separation of $M$. Since $M_{0}$ is internally 4-connected it follows that either $\left|E\left(M_{0}\right) \cap\left(E_{1}-T\right)\right| \leq 3$ or $\left|E\left(M_{0}\right) \cap\left(E_{2}-T\right)\right| \leq 3$. By relabeling if necessary we will assume the former.

Let $(A, B)$ be a partition of $E_{1}-\left(T \cup E\left(M_{0}\right)\right)$ such that $M_{0}$ is a minor of $M / A \backslash B$. We can assume that $A$ is independent in $M$. Note that as $\left|E_{1}-T\right| \geq 4$ it follows that $A \cup B$ is non-empty. Let $N=M_{1} / A \backslash B$. Proposition 2.2 says that $M / A \backslash B=N \triangle M_{2}$.

Assume that $r_{N}(T)=0$. It is easy to see that $\left(E(N)-T, E\left(M_{2}\right)-T\right)$ is a 1-separation of $M / A \backslash B$. As $M_{0}$ is internally 4-connected and $E\left(M_{0}\right) \cap$ $\left(E_{2}-T\right)$ is non-empty it follows that $E(N)-T=\varnothing$. Thus $E(N)=T$ and $N$ consists of three loops. Proposition 2.5 implies that $N \triangle M_{2}=M_{2} / T$, and as $M_{0}$ is a minor of $M / A \backslash B=N \triangle M_{2}$ it follows that $M_{2}$ has an $M_{0}$-minor, a contradiction.

Next we assume that $r_{N}(T)=1$. Since $A$ is independent in $M$ it must be independent in $M_{1}$. There is a circuit $C^{\prime} \subseteq A \cup T$ of $M_{1}$ such that $C^{\prime}$ meets both $A$ and $T$. Clearly $C^{\prime}$ cannot meet $T$ in three elements as $T$ is a triangle of $M_{1}$. Suppose that $C^{\prime}$ meets $T$ in two elements. Then $C^{\prime} \triangle T$ is a disjoint union of circuits of $M_{1}$. Thus there is a circuit $C \subseteq A \cup T$ such that $C$ meets $T$ in exactly one element. Let this element be $e$, and suppose that $T=\{e, f, g\}$. If $f \in \operatorname{cl}_{M_{1}}(A)$ then $T \subseteq \operatorname{cl}_{M_{1}}(A)$, which implies that $r_{N}(T)=0$, contrary to hypothesis. Similarly $g \notin \mathrm{cl}_{M_{1}}(A)$, so $e$ is a loop and $\{f, g\}$ is a parallel pair in $N$.

We will assume that $E(N)-T \neq \varnothing$. Assume that $\operatorname{cl}_{N}(E(N)-T)$ does not contain $\{f, g\}$. If $C$ is a circuit of $N \triangle M_{2}$ that meets both $E(N)-T$ and $E_{2}-T$ then there must be a cycle $Z$ of $N$ such that $Z-T=C \cap(E(N)-T)$. Let $C^{\prime} \subseteq Z$ be a circuit of $N$ such that $C^{\prime} \cap(E(N)-T) \neq \varnothing$. It cannot be the case that $e \in C^{\prime}$, as $e$ is a loop of $N$. Similarly, $C^{\prime}$ cannot contain both $f$ and $g$. Our assumption means that $C^{\prime}$ cannot contain precisely one of $f$ and $g$. Therefore $C^{\prime} \subseteq E(N)-T$. But this implies that $C^{\prime}$ contains a circuit of $N \triangle M_{2}$ that is properly contained in $C$, a contradiction. Therefore $\lambda_{N \triangle M_{2}}(E(N)-T)=0$, and this means that $E(N)-T$ is empty, contrary to hypothesis. Henceforth we will assume that $\{f, g\} \subseteq \operatorname{cl}_{N}(E(N)-T)$.

Let $B_{1}$ be a basis of $N$ such that $f \in B_{1}$. We show that there is no cycle $Z$ of $N$ such that $Z \subseteq B_{1} \cup T$ and $Z$ meets $B_{1}-f$. Suppose that $Z$ is such a cycle and let $C \subseteq Z$ be a circuit of $N$ such that $C \cap\left(B_{1}-f\right) \neq \varnothing$. As $B_{1}$ is independent in $N$ it follows that $C$ meets $T-f$. However $C$ cannot contain $e$ as it is a loop of $N$. Moreover $C$ cannot contain $\{f, g\}$ as it is a 
parallel pair in $N$. Thus $C \cap T=\{g\}$. But then $(C-g) \cup f$ is a circuit of $N$ contained in $B_{1}$, a contradiction.

Since $T$ is a coindependent triangle of $M_{2}$ it follows that there is a basis $B_{2}$ of $M_{2}$ such that $B_{2} \cap T=\{e\}$. The argument in the previous paragraph implies that if $\left(B_{1}-f\right) \cup\left(B_{2}-e\right)$ is dependent in $N \triangle M_{2}$, then $B_{2}-e$ must contain a circuit $C$ of $N \triangle M_{2}$. But Proposition 2.17 implies that $B_{2}-e$ is independent in $N \triangle M_{2}$. Therefore $\left(B_{1}-f\right) \cup\left(B_{2}-e\right)$ is independent in $N \triangle M_{2}$, so

$$
r(N)+r\left(M_{2}\right)-2 \leq r\left(N \triangle M_{2}\right) .
$$

Let $B$ be a basis of $N \triangle M_{2}$ restricted to $E(N)-T$. It cannot be the case that $B$ contains a circuit $C$ of $N$, for $C$ would be a cycle in $N \triangle M_{2}$. Therefore $B$ is independent in $N$, so

$$
r_{N \triangle M_{2}}(E(N)-T) \leq r_{N}(E(N)-T) \leq r(N) .
$$

Proposition 2.17 shows that $B_{2}-e$ is a basis of $E_{2}-T$ in $N \triangle M_{2}$. Thus

$$
r_{N \triangle M_{2}}\left(E_{2}-T\right)=r\left(M_{2}\right)-1 .
$$

By combining Equations (1), (2), and (3) we see that $\lambda_{N \triangle M_{2}}(E(N)-T) \leq 1$. Now we see that $E(N)-T$ contains exactly one element, $x$.

If $x$ is not parallel to $f$ and $g$ in $N$ then $x$ is a loop or a coloop in $N$, and it is easy to see that it is therefore a loop or coloop in $N \triangle M_{2}$, which leads to a contradiction. Therefore $\{f, g, x\}$ is a parallel class of $N$. Suppose that $M_{2}^{+}$is the matroid obtained from $M_{2}$ by adding an element $x^{\prime}$ in parallel to $f$. Now $\{f, x\}$ and $\left\{f, x^{\prime}\right\}$ are cycles of $N$ and $M_{2}^{+}$respectively. Thus $\left\{x, x^{\prime}\right\}$ is a cycle of $N \triangle M_{2}^{+}$and therefore either $\left\{x, x^{\prime}\right\}$ is a parallel pair, or both $x$ and $x^{\prime}$ are loops of $N \triangle M_{2}^{+}$. In either case

$$
N \triangle M_{2}=N \triangle\left(M_{2}^{+} \backslash x^{\prime}\right)=\left(N \triangle M_{2}^{+}\right) \backslash x^{\prime} \cong\left(N \triangle M_{2}^{+}\right) \backslash x=(N \backslash x) \triangle M_{2}^{+} .
$$

However Proposition 2.18 asserts that $(N \backslash x) \triangle M_{2}^{+}$is equal to $M_{2}^{+} / e \backslash f \backslash g$, which is isomorphic to $M_{2} / e \backslash g$. Thus $M_{2}$ has an $M_{0}$-minor, a contradiction.

Therefore we suppose that $E(N)-T=\varnothing$. Proposition 2.18 tells us that $N \triangle M_{2}$ is equal to $M_{2} / e \backslash f \backslash g$. As $M_{0}$ is a minor of $N \triangle M_{2}$ it follows that $M_{2}$ again has an $M_{0}$-minor, contrary to hypothesis.

We must now consider the case that $r_{N}(T)=2$. Suppose that $E(N)-T$ contains a circuit of size at most two in $N$. Then $N \backslash T$ contains a circuit of size at most two. Since $T$ is a triangle in both $N$ and $M_{2}$ it follows from Proposition 2.3 that the restriction of $N \triangle M_{2}$ to $E(N)-T$ contains a circuit of size at most two. This implies that $M_{0}$ has a circuit with at most two elements, a contradiction.

Next we similarly assume that $E(N)-T$ contains a cocircuit of size at most two in $N$. Then $N / T$ contains a cocircuit of size at most two. However, $T$ is coindependent in $M_{2}$, which means that $T$ comprises three loops in $M_{2} /\left(E\left(M_{2}\right)-T\right)$. Now Proposition 2.5 says that $N / T$ is equal to

$$
\left.N \triangle\left(M_{2} /\left(E\left(M_{2}\right)-T\right)\right)=\left(N \triangle M_{2}\right) /\left(E\left(M_{2}\right)-T\right)\right) .
$$


Thus $E(N)-T$ contains a cocircuit of size at most two in $N \triangle M_{2}$. This implies that $M_{0}$ contains a cocircuit of size at most two, a contradiction.

We have shown that every circuit and cocircuit of $N$ that is contained in $E(N)-T$ has size at least three. As $|E(N)-T| \leq 3$ it is now easy to see that either $N$ is isomorphic to $M\left(K_{4}\right)$, or $r(N)=2$ and $N$ contains exactly three parallel classes, each one of size at most two. Suppose that $N$ is isomorphic to $M\left(K_{4}\right)$. In this case $N \triangle M_{2}$ is equal to $\Delta_{T}\left(M_{2}\right)$, so $\Delta_{T}\left(M_{2}\right)$ has an $M_{0}$-minor. Moreover $T$ is a triad of $\Delta_{T}\left(M_{2}\right)$ by Proposition 2.6. As $T \subseteq E\left(M_{0}\right)$ and $M_{0}$ has no cocircuits of size less than three it follows that $M_{0}$ has at least one triad, as desired.

Finally we assume that every element in $E(N)-T$ is parallel to an element of $T$ in $N$, and that $N$ contains no parallel class of more than two elements. By using the same arguments as before we see that we can replace every element in $N$ that is parallel to an element in $T$ with an element in $M_{2}$ that is parallel to the same member of $T$. Thus we can assume that $E(N)=T$. Now it follows easily from Proposition 2.3 that $N \triangle M_{2}$ is isomorphic to a minor of $M_{2}$. Thus $M_{2}$ has an $M_{0}$-minor, a contradiction. This completes the proof of the lemma.

2.5. Möbius matroids. In this section we describe the Möbius matroids. The cubic Möbius ladder $C M_{2 n}$ is the cubic graph obtained from an even cycle with vertices $v_{0}, \ldots, v_{2 n-1}$ by joining each vertex $v_{i}$ to the antipodal vertex $v_{i+n}$. (Indices are to be read modulo $2 n$.) The quartic Möbius lad$\operatorname{der} Q M_{2 n+1}$ is the quartic graph obtained from an odd cycle with vertices $v_{0}, \ldots, v_{2 n}$ by joining each vertex $v_{i}$ to the two antipodal vertices $v_{i+n}$ and $v_{i+n+1}$. (In this case indices are read modulo $2 n+1$.) In either case, the edges of the cycle are known as rim edges, and the diagonal edges are known as spokes.

Triangular Möbius matroids. Let $r$ be an integer exceeding two and let $\left\{e_{1}, \ldots, e_{r}\right\}$ be the standard basis in the vector space of dimension $r$ over $\mathrm{GF}(2)$. For $1 \leq i \leq r-1$ let $a_{i}$ be the sum of $e_{i}$ and $e_{r}$, and for $1 \leq i \leq r-2$ let $b_{i}$ be the sum of $e_{i}$ and $e_{i+1}$. Let $b_{r-1}$ be the sum of $e_{1}, e_{r-1}$, and $e_{r}$. The rank-r triangular Möbius matroid, denoted by $\Delta_{r}$, is represented over $\mathrm{GF}(2)$ by the set $\left\{e_{1}, \ldots, e_{r}, a_{1}, \ldots, a_{r-1}, b_{1}, \ldots, b_{r-1}\right\}$. Thus $\Delta_{r}$ has rank $r$ and $\left|E\left(\Delta_{r}\right)\right|=3 r-2$. Deleting $e_{r}$ from $\Delta_{r}$ produces a matroid isomorphic to the bond matroid of a cubic Möbius ladder. We say that $a_{1}, \ldots, a_{r-1}$ and $e_{1}, \ldots, e_{r-1}$ are the rim elements of $\Delta_{r}$, and that $b_{1}, \ldots, b_{r-1}$ are the spoke elements. It is easy to see that if $r \geq 4$, then $\Delta_{r}$ has $\Delta_{r-1}$ as a minor.

Triadic Möbius matroids. Let $r \geq 4$ be an even integer, and again let $\left\{e_{1}, \ldots, e_{r}\right\}$ be the standard basis of the vector space over $\mathrm{GF}(2)$ of dimension $r$. For $1 \leq i \leq r-2$ let $c_{i}$ be the sum of $e_{i}, e_{i+1}$, and $e_{r}$. Let $c_{r-1}$ be the sum of $e_{1}, e_{r-1}$, and $e_{r}$. The rank-r triadic Möbius matroid, denoted by $\Upsilon_{r}$, is represented over $\operatorname{GF}(2)$ by the set $\left\{e_{1}, \ldots, e_{r}, c_{1}, \ldots, c_{r-1}\right\}$. Thus $\Upsilon_{r}$ has rank $r$ and $\left|E\left(\Upsilon_{r}\right)\right|=2 r-1$. If $r \geq 4$ is an even integer then $\Upsilon_{r} \backslash e_{r}$ 
is isomorphic to the bond matroid of a quartic Möbius ladder. We say that $e_{1}, \ldots, e_{r-1}$ are the rim elements of $\Upsilon_{r}$ and $c_{1}, \ldots, c_{r-1}$ are spoke elements. If $r$ is an even integer greater than 4 , then $\Upsilon_{r}$ has $\Upsilon_{r-2}$ as a minor.

\section{OTHER CLASSES OF BINARY MATROIDS}

Recall that if $\mathcal{M}$ is a set of binary matroids, then $\mathcal{E X}(\mathcal{M})$ is the class of binary matroids that have no minors in $\mathcal{M}$. Let $\mathcal{M}$ be a subset of the collection

$$
\left\{M\left(K_{3,3}\right), M^{*}\left(K_{3,3}\right), M\left(K_{5}\right), M^{*}\left(K_{5}\right)\right\}
$$

with the property that $\mathcal{M}$ contains either $M\left(K_{3,3}\right)$ or its dual. There are exactly twelve classes of binary matroids of the form $\mathcal{E} \mathcal{X}(\mathcal{M})$. Theorem 1.1, and the famous graph-theoretical results of Hall [6] and Wagner [23], lead to characterizations of the internally 4-connected matroids in each of these classes. The first such characterization is given by Theorem 1.1.

Lemma 3.1. The triangular and triadic Möbius matroids have no $M\left(K_{5}\right)$-minors.

Proof. Lemma 3.8 of [12] states that the only internally 4-connected noncographic minors of Möbius matroids are themselves Möbius matroids. Thus if a Möbius matroid had an $M\left(K_{5}\right)$-minor it would imply that $M\left(K_{5}\right)$ is a Möbius matroid. But the only rank-4 Möbius matroid with a ground set of size ten is $\Delta_{4}$, and $\Delta_{4}$ has only nine triangles. Thus no Möbius matroid has an $M\left(K_{5}\right)$-minor.

The next result follows from Lemma 3.1 and a simple computer check.

Theorem 3.2. An internally 4-connected binary matroid $M$ has no $M\left(K_{3,3}\right)$-minor and no $M\left(K_{5}\right)$-minor if and only if $M$ is either:

(i) cographic;

(ii) isomorphic to $\Delta_{r}$ for some integer $r \geq 3$ or to $\Upsilon_{r}$ for some even integer $r \geq 4 ;$ or

(iii) isomorphic to one of the following sporadic matroids: $C_{11}, C_{12}, M_{5,12}^{a}$, $M_{6,13}, M_{7,15}, M_{9,18}$, or $M_{11,21}$.

Wagner [23] characterized the graphs with no $K_{5}$-minor (see also [9, Theorem 1.6].) The following matroidal corollary of his theorem is well known, although its proof seems not to appear in the literature.

Lemma 3.3. If $M$ is an internally 4-connected cographic matroid with no minor isomorphic to $M^{*}\left(K_{5}\right)$ then either $M=M^{*}(G)$, where $G$ is a planar graph, or $M$ is isomorphic to one of $M^{*}\left(K_{3,3}\right)$ or $M^{*}\left(C M_{8}\right)$.

It is easy to check using a computer that $\Delta_{6}$ has an $M^{*}\left(K_{5}\right)$-minor, and therefore $\Delta_{r}$ has an $M^{*}\left(K_{5}\right)$-minor for all $r \geq 6$. On the other hand $\Delta_{r}$ has no $M^{*}\left(K_{5}\right)$-minor if $r \in\{3,4,5\}$. Similarly $\Upsilon_{r}$ has an $M^{*}\left(K_{5}\right)$-minor if $r \geq 6$, but $\Upsilon_{4}$ has no $M^{*}\left(K_{5}\right)$-minor. We note that $\Delta_{3} \cong F_{7}$ and $\Upsilon_{4} \cong F_{7}^{*}$. The next theorem follows from these facts, and by applying Theorem 1.1, Lemma 3.3, and some computer tests. 
Theorem 3.4. An internally 4-connected binary matroid $M$ has no $M\left(K_{3,3}\right)$-minor and no $M^{*}\left(K_{5}\right)$-minor if and only if $M$ is either:

(i) planar graphic;

(ii) isomorphic to one of the cographic matroids $M^{*}\left(K_{3,3}\right)$ or $M^{*}\left(C M_{8}\right)$;

(iii) isomorphic to one of the following Möbius matroids: $F_{7}, F_{7}^{*}, \Delta_{4}, \Delta_{5}$; or,

(iv) isomorphic to one of the 18 sporadic matroids of Theorem 1.1, other than $T_{12}$.

A result due to Hall [6] implies that the only 3-connected cographic matroids with no $M^{*}\left(K_{3,3}\right)$-minor are $M^{*}\left(K_{5}\right)$, and cycle matroids of planar graphs. The only Möbius matroids with no $M^{*}\left(K_{3,3}\right)$-minor are $\Delta_{3} \cong F_{7}$, $\Upsilon_{4} \cong F_{7}^{*}$, and $\Upsilon_{6}$. Corollary 2.15 of [12] says that the only internally 4-connected binary matroids that are non-cographic and have no minor isomorphic to either $M\left(K_{3,3}\right)$ or $\Delta_{4}$ are $F_{7}, F_{7}^{*}, M\left(K_{5}\right), T_{12} \backslash e, T_{12} / e$, and $T_{12}$. A computer check reveals that none of these matroids has an $M^{*}\left(K_{3,3}\right)$-minor. Both $T_{12}$ and $T_{12} / e$ are among the sporadic matroids of Theorem 1.1, while $T_{12} \backslash e \cong \Upsilon_{6}$. Since $\Delta_{4}$ has an $M^{*}\left(K_{3,3}\right)$-minor, the next result follows.

Theorem 3.5. An internally 4-connected binary matroid $M$ has no $M\left(K_{3,3}\right)$-minor and no $M^{*}\left(K_{3,3}\right)$-minor if and only if $M$ is either:

(i) planar graphic;

(ii) isomorphic to the cographic matroid $M^{*}\left(K_{5}\right)$;

(iii) isomorphic to one of the following Möbius matroids: $F_{7}, F_{7}^{*}$, or $\Upsilon_{6}$; or,

(iv) isomorphic to one of the following sporadic matroids: $M\left(K_{5}\right), T_{12} / e$, or $T_{12}$.

The next theorems are easy consequences of results stated above.

Theorem 3.6. An internally 4-connected binary matroid $M$ belongs to $\mathcal{E X}\left(M\left(K_{3,3}\right), M\left(K_{5}\right), M^{*}\left(K_{5}\right)\right)$ if and only if $M$ is either:

(i) planar graphic;

(ii) isomorphic to one of the cographic matroids $M^{*}\left(K_{3,3}\right)$ or $M^{*}\left(C M_{8}\right)$;

(iii) isomorphic to one of the following Möbius matroids: $F_{7}, F_{7}^{*}, \Delta_{4}, \Delta_{5}$; or,

(iv) isomorphic to one of the following sporadic matroids: $C_{11}, C_{12}, M_{5,12}^{a}$, $M_{6,13}, M_{7,15}, M_{9,18}$, or $M_{11,21}$.

Theorem 3.7. An internally 4-connected binary matroid $M$ belongs to $\mathcal{E} \mathcal{X}\left(M\left(K_{3,3}\right), M^{*}\left(K_{3,3}\right), M\left(K_{5}\right)\right)$ if and only if $M$ is either:

(i) planar graphic;

(ii) isomorphic to the cographic matroid $M^{*}\left(K_{5}\right)$; or,

(iii) isomorphic to one of the following Möbius matroids: $F_{7}, F_{7}^{*}$, or $\Upsilon_{6}$.

Finally, we have the following characterization, which has already been proved by Qin and Zhou [16]. 
Theorem 3.8. An internally 4-connected binary matroid $M$ belongs to $\mathcal{E X}\left(M\left(K_{3,3}\right), M^{*}\left(K_{3,3}\right), M\left(K_{5}\right), M^{*}\left(K_{5}\right)\right)$ if and only if $M$ is either:

(i) planar graphic; or,

(ii) isomorphic to one of $F_{7}$ or $F_{7}^{*}$.

By dualizing Theorems 1.1, 3.2, 3.4, 3.6, and 3.7, we obtain five additional characterizations of classes.

\section{Polynomial-time algorithms}

In this section we show that our structural characterizations lead to polynomial-time algorithms for deciding membership in each of the twelve classes of binary matroids described in Section 3; at least as long as the input consists of a matrix over $\mathrm{GF}(2)$. We will set aside consideration of oracle algorithms for the moment and return to them later.

4.1. Algorithms for binary matroids. For now we assume that a binary matroid on a ground set of size $n$ is described by a matrix over GF(2) with $n$ columns. We can assume that such a matrix has no more than $n$ rows. The next result, due to Cunningham and Edmonds (see [1, Section 6.5.3]), relies upon the matroid intersection algorithm of Edmonds [4].

Proposition 4.1. For each $k \in\{1,2\}$ there is a polynomial-time algorithm that will either output a $k$-separation of a binary matroid $M$, or decide that $M$ has no such separation. Moreover there is a polynomial-time algorithm that, for a binary matroid $M$, will either output a 3-separation $\left(X_{1}, X_{2}\right)$ of $M$ such that $\left|X_{1}\right|,\left|X_{2}\right| \geq 4$, or decide that no such separation exists.

If $M$ is a rank- $r$ binary matroid with no loops, then we can consider $M$ as a multiset of points in the projective space $P=\mathrm{PG}(r-1,2)$. If $X \subseteq E(M)$, then $X$ is represented by a multiset of points in $P$, and we use $\operatorname{cl}_{P}(X)$ to denote the span of this set in $P$. The next result is well known, al beit difficult to find in the literature.

Proposition 4.2. Let $M$ be a binary matroid of rank $r$, and let $P=$ $\operatorname{PG}(r-1,2)$. Suppose that $\left(X_{1}, X_{2}\right)$ is an exact $k$-separation of $M$ for some $k \in\{1,2,3\}$ with the property that if $k=3$ then $\left|X_{1}\right|,\left|X_{2}\right| \geq 4$ and $r_{M}\left(X_{1}\right), r_{M}\left(X_{2}\right) \geq 3$. Let $Z=\operatorname{cl}_{P}\left(X_{1}\right) \cap \operatorname{cl}_{P}\left(X_{2}\right)$, and for $i=1$, 2 let $M_{i}$ be the binary matroid represented by the multiset $X_{i} \cup Z$. Then $M \cong M_{1} \oplus_{k} M_{2}$.

The next result is an easy consequence of Proposition 4.2.

Corollary 4.3. There is a polynomial-time algorithm that, given a representation of a loopless binary matroid $M$ and a partition $\left(E\left(M_{1}\right)\right.$ $\left.E\left(M_{2}\right), E\left(M_{2}\right)-E\left(M_{1}\right)\right)$ of $E(M)$, where $M$ is the k-sum of binary matroids $M_{1}$ and $M_{2}$ for some $k \in\{1,2,3\}$, will output representations of $M_{1}$ and $M_{2}$. 
The central idea of Seymour's algorithm for recognizing regular matroids, and of our algorithms, is that a binary matroid can be decomposed into internally 4-connected components. We now make this idea more formal.

Suppose that $M$ is a binary matroid such that $\operatorname{si}(M)$ is 3 -connected. Let $\mathcal{T}$ be a set of pairwise disjoint triangles in $M$. We recursively define a rooted tree, called a decomposition tree of $(M, \mathcal{T})$ (or just a decomposition tree of $M)$, denoted by $\Phi(M, \mathcal{T})$. Each node is labeled with a matroid and a set of disjoint triangles of that matroid. Each node of $\Phi(M, \mathcal{T})$ has indegree one, apart from the root, which has indegree zero. Moreover each node has outdegree either zero or two. Those vertices with outdegree zero are called leaves.

If $\operatorname{si}(M)$ is internally 4 -connected then $\Phi(M, \mathcal{T})$ comprises a single node: the root, which is labeled $(M, \mathcal{T})$. If $\operatorname{si}(M)$ is not internally 4-connected then there is an exact 3-separation $\left(Y_{1}, Y_{2}\right)$ of $\operatorname{si}(M)$ such that $\left|Y_{1}\right|,\left|Y_{2}\right| \geq 4$. This naturally induces a separation of $M$, as follows: Recall that the ground set of $\operatorname{si}(M)$ is the set of parallel classes of $M$. Let $\left(X_{1}, X_{2}\right)$ be the partition of $E(M)$ defined so that $x \in X_{1}$ if and only if $\operatorname{cl}_{M}(\{x\}) \in Y_{1}$. Thus $\left(X_{1}, X_{2}\right)$ is an exact 3 -separation of $M$, and $\left|X_{1}\right|,\left|X_{2}\right| \geq 4$. Let $r=r(M)$ and let $T=\operatorname{cl}_{P}\left(X_{1}\right) \cap \operatorname{cl}_{P}\left(X_{2}\right)$, where $P=\mathrm{PG}(r-1,2)$. Thus if $M_{i}=P \mid\left(X_{i} \cup T\right)$ for $i=1,2$, then $T$ is a triangle of both $M_{1}$ and $M_{2}$, and $M=M_{1} \oplus_{3} M_{2}$, by Proposition 4.2.

We want each of the triangles in $\mathcal{T}$ to be contained in either $X_{1}$ or $X_{2}$. If a triangle $T \in \mathcal{T}$ contains elements from both $X_{1}$ and $X_{2}$, then, up to relabeling, $T$ contains exactly one element of $X_{2}$. We shift this element into $X_{1}$, and add a parallel element to take its place in $X_{2}$. More precisely: Suppose that $T_{1}, \ldots, T_{p}$ is the list of triangles in $\mathcal{T}$ that are contained in neither $X_{1}$ nor $X_{2}$. We make the following assignments. Let $M^{(0)}=M$. For $i=1,2$ let $M_{i}^{(0)}=M_{i}$ and let $X_{i}^{(0)}=X_{i}$. For $k=1, \ldots, p$, let $\{i, j\}=$ $\{1,2\}$, and assume that exactly one element, $e$, of $T_{k}$ is contained in $X_{i}^{(k-1)}$. We obtain $M^{(k)}$ by adding a new element $e_{k}$ to $M^{(k-1)}$ so that it is parallel to $e$. We let $X_{i}^{(k)}=\left(X_{i}^{(k-1)}-e\right) \cup e_{k}$, and we let $X_{j}^{(k)}=X_{j}^{(k-1)} \cup e$. Since $e \in \mathrm{cl}_{M^{(k-1)}}\left(X_{1}^{(k-1)}\right) \cap \mathrm{cl}_{M^{(k-1)}}\left(X_{2}^{(k-1)}\right)$ it follows that $e$ must be parallel to an element $t$ of $T$ in $M_{i}^{(k-1)}$. We obtain $M_{i}^{(k)}$ from $M_{i}^{(k-1)}$ by relabeling the element $e$ with $e_{k}$ and we obtain $M_{j}^{(k)}$ from $M_{j}^{(k-1)}$ by adding $e$ parallel to $t$. Note that $\left(X_{1}^{(k)}, X_{2}^{(k)}\right)$ is an exact 3-separation of $M^{(k)}$, and that the number of rank-one flats in both $X_{1}^{(k)}$ and $X_{2}^{(k)}$ is at least four. It is easy to see that $M^{(k)}=M_{1}^{(k)} \oplus_{3} M_{2}^{(k)}$.

Let $M^{+}=M^{(p)}$, and for $i=1,2$ let $M_{i}^{+}=M_{i}^{(p)}$. Thus $\operatorname{si}\left(M^{+}\right)=\operatorname{si}(M)$, and $M^{+}=M_{1}^{+} \oplus_{3} M_{2}^{+}$. Moreover $\operatorname{si}\left(M_{i}^{+}\right)=\operatorname{si}\left(M_{i}\right)$ for $i=1$, 2. Furthermore every triangle in $\mathcal{T}$ is contained in either $X_{1}^{(p)}$ or $X_{2}^{(p)}$. We let $\left(\mathcal{T}_{1}, \mathcal{T}_{2}\right)$ be the partition of $\mathcal{T}$ induced by $\left(X_{1}^{(p)}, X_{2}^{(p)}\right)$. 
Note that both $\operatorname{si}\left(M_{1}^{+}\right)$and $\operatorname{si}\left(M_{2}^{+}\right)$are 3 -connected by Proposition 2.14 . We recursively define $\Phi(M, \mathcal{T})$ to be the decomposition tree obtained by starting with the root, labeled $(M, \mathcal{T})$, adding two new vertices labeled by $\left(M_{1}^{+}, \mathcal{T}_{1} \cup\{T\}\right)$ and $\left(M_{2}^{+}, \mathcal{T}_{2} \cup\{T\}\right)$, adding arcs from the root to these two vertices, and then identifying the new vertices with the roots of $\Phi\left(M_{1}^{+}, \mathcal{T}_{1} \cup\right.$ $\{T\})$ and $\Phi\left(M_{2}^{+}, \mathcal{T}_{2} \cup\{T\}\right)$ respectively.

Observe that, as $M_{1}^{+}$and $M_{2}^{+}$have strictly fewer rank-one flats than $M$, it must be the case that this recursive procedure will eventually terminate. Thus the leaves of $\Phi(M, \mathcal{T})$ are labeled with pairs $\left(M_{1}, \mathcal{T}_{1}\right), \ldots,\left(M_{p}, \mathcal{T}_{p}\right)$ such that $\operatorname{si}\left(M_{i}\right)$ is internally 4 -connected for all $i \in\{1, \ldots, p\}$, and $\mathcal{T}_{i}$ is a set of pairwise disjoint triangles in $M_{i}$. Note that $\Phi(M, \mathcal{T})$ need not be unique: rather it depends upon our choice of 3-separations.

Proposition 4.4. Suppose that $M$ is a binary matroid such that $\operatorname{si}(M)$ is 3-connected and that $\mathcal{T}$ is a set of disjoint triangles of $M$. Let $n$ be the number of rank-one flats of $M$. Then the number of leaves in $\Phi(M, \mathcal{T})$ is at most $\max \{1, n-6\}$.

Proof. The proof is by induction on $n$. If $n \leq 7$ then $\operatorname{si}(M)$ is internally 4-connected, so $\Phi(M, \mathcal{T})$ has one leaf and we are done. Thus assume that $n>7$ and that $\operatorname{si}(M)$ is not internally 4-connected. Suppose that the children of $M$ in $\Phi(M, \mathcal{T})$ are labeled $\left(M_{1}^{+}, \mathcal{T}_{1} \cup\{T\}\right)$ and $\left(M_{2}^{+}, \mathcal{T}_{2} \cup\{T\}\right)$ respectively. For $i=1,2$ let the number of rank-one flats in $M_{i}^{+}$be $n_{i}$. Then $n_{1}+n_{2} \leq n+3$. Since $n_{1}, n_{2}<n$ the inductive hypothesis implies that $\Phi\left(M_{1}^{+}, \mathcal{T}_{1} \cup\{T\}\right)$ and $\Phi\left(M_{2}^{+}, \mathcal{T}_{2} \cup\{T\}\right)$ have at $\operatorname{most} \max \left\{1, n_{1}-6\right\}$ and $\max \left\{1, n_{2}-6\right\}$ leaves respectively. The result follows easily.

Proposition 4.5. There is a polynomial-time algorithm which, given a binary matroid $M$ with the property that $\operatorname{si}(M)$ is 3-connected, and a set $\mathcal{T}$ of pairwise disjoint triangles of $M$, will compute a decomposition tree $\Phi(M, \mathcal{T})$.

Proof. Let $n=E(M)$. We can assume that $n \geq 7$. Note that $|\mathcal{T}| \leq n / 3$. Proposition 4.4 implies that the number of leaves in any decomposition tree of $M$ is at most $n-6$. Thus $\Phi(M, \mathcal{T})$ has at most $n-7$ non-leaf vertices. It follows that if a node of $\Phi(M, \mathcal{T})$ is labeled with $\left(M_{i}, \mathcal{T}_{i}\right)$ then $\left|\mathcal{T}_{i}\right| \leq 4 n / 3-$ 7. Each triangle in $\mathcal{T}_{i}$ contributes at most one extra element to the matroids which label the children of $\left(M_{i}, \mathcal{T}_{i}\right)$. It follows that if $\left(M_{j}, \mathcal{T}_{j}\right)$ is a node label then $M_{j}$ has at most $n+(4 n / 3-7)(n-7)$ elements. Proposition 4.1 and Corollary 4.3 imply the existence of a polynomial-time algorithm which finds the two terms of each decomposition along a 3-sum. Suppose that this algorithm runs in time bounded by $n^{k}$ for an $n$-element matroid, where $k$ is a fixed constant. Clearly, given a representation of an $n$-element matroid, it is possible to construct a representation of the simplification of that matroid in time bounded by $n^{2}$. It follows that there is an algorithm that constructs $\Phi(M, \mathcal{T})$ in time bounded by

$$
(n-7)(n+(4 n / 3-7)(n-7))^{k+2} .
$$


Proposition 4.6. Let $M_{0}$ be a simple binary matroid. Suppose that $M$ is a binary matroid such that $\operatorname{si}(M)$ is 3 -connected and that $\mathcal{T}$ is a set of pairwise disjoint triangles of $M$. Let $\Phi(M, \mathcal{T})$ be a decomposition tree of $M$. If there is some leaf of $\Phi(M, \mathcal{T})$ labeled $\left(M_{i}, \mathcal{T}_{i}\right)$, where $M_{i}$ has an $M_{0}$-minor, then $M$ has an $M_{0}$-minor.

Proof. The proof is by induction on the number of vertices in $\Phi(M, \mathcal{T})$. If $\Phi(M, \mathcal{T})$ has only one node then $M_{i}$ must be equal to $M$ and the result is trivial. Suppose that $\Phi(M, \mathcal{T})$ has more than one node, and suppose that the children of $(M, \mathcal{T})$ are labeled with $\left(M_{1}^{+}, \mathcal{T}_{1} \cup\{T\}\right)$ and $\left(M_{2}^{+}, \mathcal{T}_{2} \cup\{T\}\right)$. Without loss of generality we can assume that the leaf labeled by $\left(M_{i}, \mathcal{T}_{i}\right)$ belongs to $\Phi\left(M_{1}^{+}, \mathcal{T}_{1} \cup\{T\}\right)$. By induction $M_{1}^{+}$has an $M_{0}$-minor. Proposition 2.13 says that $M^{+}=M_{1}^{+} \oplus_{3} M_{2}^{+}$has an $M_{0}$-minor. But $\operatorname{si}\left(M^{+}\right)=\operatorname{si}(M)$, and as $M_{0}$ is simple it follows that $M$ has an $M_{0}$-minor.

Proposition 4.7. Suppose that $M_{0}$ is an internally 4-connected binary matroid such that $\left|E\left(M_{0}\right)\right| \geq 4$ and $M_{0}$ has no triads. Suppose also that $M$ is a binary matroid such that $\operatorname{si}(M)$ is 3-connected, and that $\mathcal{T}$ is a collection of pairwise disjoint triangles of $M$. Let $\Phi(M, \mathcal{T})$ be a decomposition tree of $M$. If $M$ has an $M_{0}$-minor then there is a leaf of $\Phi(M, \mathcal{T})$ labeled $\left(M_{i}, \mathcal{T}_{i}\right)$ such that $M_{i}$ has an $M_{0}$-minor.

Proof. The proof is by induction on the number of vertices in $\Phi(M, \mathcal{T})$. If $\Phi(M, \mathcal{T})$ has only one node the result is obvious. Suppose that $\Phi(M, \mathcal{T})$ has more than one node and assume that the children of $(M, \mathcal{T})$ are $\left(M_{1}^{+}, \mathcal{T}_{1} \cup\right.$ $\{T\})$ and $\left(M_{2}^{+}, \mathcal{T}_{2} \cup\{T\}\right)$. If either $M_{1}^{+}$or $M_{2}^{+}$has an $M_{0}$-minor then the result follows by induction. Therefore we assume that neither $M_{1}^{+}$nor $M_{2}^{+}$has an $M_{0}$-minor. However, if $M^{+}=M_{1}^{+} \oplus_{3} M_{2}^{+}$then $\operatorname{si}\left(M^{+}\right) \cong M$. Therefore $M^{+}$has an $M_{0}$-minor. Lemma 2.19 implies that $M_{0}$ contains at least one triad, a contradiction.

Suppose that $M$ is a binary matroid and that $\mathcal{T}$ is a set of pairwise disjoint triangles of $M$. We define $\Delta(M ; \mathcal{T})$ to be the matroid produced by performing $\Delta-Y$ operations on each of the triangles in $\mathcal{T}$. Proposition 2.8 tells us that $\Delta(M ; \mathcal{T})$ is well-defined. The next result follows from repeated application of Proposition 2.16.

Proposition 4.8. Suppose that $M_{1}$ and $M_{2}$ are binary matroids and that the 3-sum of $M_{1}$ and $M_{2}$ along the triangle $T$ is defined. For $i=1,2$ let $\mathcal{T}_{i}$ be a set of pairwise disjoint triangles of $M_{i}$ that do not meet $T$. Then

$$
\Delta\left(M_{1} ; \mathcal{T}_{1}\right) \oplus_{3} \Delta\left(M_{2} ; \mathcal{T}_{2}\right)=\Delta\left(M_{1} \oplus_{3} M_{2} ; \mathcal{T}_{1} \cup \mathcal{T}_{2}\right) .
$$

Proposition 4.9. Let $M_{0}$ be a 3-connected binary matroid such that $\left|E\left(M_{0}\right)\right| \geq 4$ and $M_{0}$ has no triangles. Suppose that $M$ is a binary matroid and that $\mathcal{T}$ is a collection of pairwise disjoint triangles of $M$. Suppose that the element $e$ is in a parallel pair in $M$. If $\Delta(M ; \mathcal{T})$ has an $M_{0}$-minor then $\Delta(M ; \mathcal{T}) \backslash$ e has an $M_{0}$-minor. 
Proof. The proof is by induction on the number of triangles in $\mathcal{T}$. If $\mathcal{T}$ is empty then the result is obvious, as $M_{0}$ has no parallel pairs. Suppose that $T_{1}, \ldots, T_{p}$ are the triangles in $\mathcal{T}$. Define $M^{(0)}$ to be $M$, and for $i=1, \ldots, p$ define $M^{(i)}$ to be $\Delta_{T_{i}}\left(M^{(i-1)}\right)$. Thus $M^{(p)}=\Delta(M ; \mathcal{T})$. If $e$ is in a parallel pair in $M^{(1)}$ then the result follows by the inductive hypothesis, as $M^{(p)}=$ $\Delta\left(M^{(1)} ; \mathcal{T}-\left\{T_{1}\right\}\right)$. Therefore we will assume that $e$ is not in a parallel pair in $M^{(1)}$. Since $e$ is in a parallel pair in $M^{(0)}$ we conclude that $e$ is parallel to an element $t$ of $T_{1}$ in $M^{(0)}$. If $p>1$ then Proposition 2.7 implies that $\{e, t\}$ is a parallel pair in $\Delta_{T_{2}}(M)$. Using Proposition 2.8 we can apply the inductive hypothesis to $M^{(p)}=\Delta\left(\Delta_{T_{2}}(M) ; \mathcal{T}-\left\{T_{2}\right\}\right)$ and conclude that the result holds. Therefore we will assume that $p=1$.

Note that $\{e, t\}$ is a cycle of $M$. It is easy to see that $\left(T_{1}-t\right) \cup e$ is a triangle of $\Delta_{T_{1}}(M)$. Let $T_{1}-t=\left\{t_{0}, t_{1}\right\}$. Suppose that $\Delta_{T_{1}}(M) / t_{0}$ has an $M_{0}$-minor. As $\left\{e, t_{1}\right\}$ is a parallel pair in $\Delta_{T_{1}}(M) / t_{0}$ it follows that $\Delta_{T_{1}}(M) / t_{0} \backslash e$ has an $M_{0}$-minor, and we are done. Therefore we will assume that $\Delta_{T_{1}}(M) / t_{0}$ has no minor isomorphic to $M_{0}$, and (by the same argument), neither does $\Delta_{T_{1}}(M) / t_{1}$.

If $t_{0}$ is in a series pair of $\Delta_{T_{1}}(M)$ then $\Delta_{T_{1}}(M) / t_{0}$ must have an $M_{0}$-minor, contrary to our assumption. Therefore we assume that neither $t_{0}$ nor $t_{1}$ (by the same argument) is in a series pair in $\Delta_{T_{1}}(M)$. Propositions 2.7 and 2.10 imply that $T_{1}$ contains a cocircuit of $\Delta_{T_{1}}(M)$. Clearly neither $t_{0}$ nor $t_{1}$ is a coloop of $\Delta_{T_{1}}(M)$, so we conclude that either $t$ is a coloop or $T_{1}$ is a triad in $\Delta_{T_{1}}(M)$. Suppose that the former holds. If there were a circuit of $M$ which met $T_{1}$ in exactly $t_{0}$ or $t_{1}$ then we could find a circuit of $\Delta_{T_{1}}(M)$ which contained $t$. Therefore no such circuit exists. It follows that $\left\{t_{0}, t_{1}\right\}$ is a series pair in $M$. As $\left\{e, t_{0}, t_{1}\right\}$ is a triangle of $M \backslash t$ it follows that $\left\{t_{0}, t_{1}\right\}$ is also a series pair in $M \backslash t$, which is equal to $\Delta_{T_{1}}(M) / t$ by Proposition 2.9. Thus $\left\{t_{0}, t_{1}\right\}$ is a series pair of $\Delta_{T_{1}}(M)$, contrary to our conclusion. Therefore $T_{1}$ is a triad of $\Delta_{T_{1}}(M)$.

As $M_{0}$ contains no triangles we must delete an element of $\left(T_{1}-t\right) \cup e$ from $\Delta_{T_{1}}(M)$ to obtain an $M_{0}$-minor. If this element is $e$ then we are done, so assume that it is $t_{0}$ (the case when it is $t_{1}$ is identical). But $\left\{t, t_{1}\right\}$ is a series pair of $\Delta_{T_{1}}(M) \backslash t_{0}$, so $\Delta_{T_{1}}(M) \backslash t_{0} / t_{1}$, and hence $\Delta_{T_{1}}(M) / t_{1}$ has an $M_{0}$-minor, contrary to our earlier conclusion. This completes the proof.

Lemma 4.10. Suppose that $M_{0}$ is an internally 4-connected binary matroid such that $\left|E\left(M_{0}\right)\right| \geq 4$ and $M_{0}$ has no triangles. Suppose also that $M$ is a binary matroid such that $\operatorname{si}(M)$ is 3-connected, and that $\mathcal{T}$ is a collection of pairwise disjoint triangles of $M$. Let $\Phi(M, \mathcal{T})$ be a decomposition tree of $M$. If $M$ has an $M_{0}$-minor then there is a leaf of $\Phi(M, \mathcal{T})$ labeled $\left(M_{i}, \mathcal{T}_{i}\right)$ with the property that $\Delta\left(M_{i} ; \mathcal{T}_{i}\right)$ has an $M_{0}$-minor.

Proof. Let $\mathcal{T}=\left\{T_{1}, \ldots, T_{p}\right\}$. Define $M^{(0)}$ to be $M$, and for $i \in\{1, \ldots, p\}$ let $M^{(i)}$ be $\Delta_{T_{i}}\left(M^{(i-1)}\right)$. Thus $M^{(p)}=\Delta(M ; \mathcal{T})$. We start by showing that $\Delta(M ; \mathcal{T})$ has an $M_{0}$-minor. If this is not the case there is some $i \in\{1, \ldots, p\}$ such that $M^{(i-1)}$ has an $M_{0}$-minor but $M^{(i)}$ does not. Since $T_{i}$ is a triangle 
of $M^{(i-1)}$ and $M_{0}$ has no triangles it follows that there is an element $a \in T_{i}$ such that $M^{(i-1)} \backslash a$ has an $M_{0}$-minor. But Proposition 2.9 says that

$$
M^{(i)} / a=\Delta_{T_{i}}\left(M^{(i-1)}\right) / a=M^{(i-1)} \backslash a .
$$

Therefore $M^{(i)}$ has an $M_{0}$-minor, a contradiction.

Suppose that the lemma is false. The argument in the previous paragraph shows that there is at least one node $\left(M_{i}, \mathcal{T}_{i}\right)$ such that $\Delta\left(M_{i} ; \mathcal{T}_{i}\right)$ has an $M_{0}$-minor. Suppose that $\left(M_{i}, \mathcal{T}_{i}\right)$ has been chosen so that if $\left(M_{j}, \mathcal{T}_{j}\right)$ is a descendant of $\left(M_{i}, \mathcal{T}_{i}\right)$ then $\Delta\left(M_{j} ; \mathcal{T}_{j}\right)$ does not have an $M_{0}$-minor. Since the lemma is false $\left(M_{i}, \mathcal{T}_{i}\right)$ cannot be a leaf node, so it has two children. Let us suppose that they are labeled $\left(M_{1}^{+}, \mathcal{T}_{1} \cup\{T\}\right)$ and $\left(M_{2}^{+}, \mathcal{T}_{2} \cup\{T\}\right)$.

Assume that $\Delta\left(M_{j}^{+} ; \mathcal{T}_{j}\right)$ has an $M_{0}$-minor, for some $j \in\{1,2\}$. Repeated use of Proposition 2.3 tells us that $T$ is a triangle of $\Delta\left(M_{j}^{+} ; \mathcal{T}_{j}\right)$. Since $M_{0}$ has no triangles there must be an element $a \in T$ such that $\Delta\left(M_{j}^{+} ; \mathcal{T}_{j}\right) \backslash a$ has an $M_{0}$-minor. But

$$
\Delta\left(M_{j}^{+} ; \mathcal{T}_{j}\right) \backslash a=\Delta_{T}\left(\Delta\left(M_{j}^{+} ; \mathcal{T}_{j}\right)\right) / a=\Delta\left(M_{j}^{+} ; \mathcal{T}_{j} \cup\{T\}\right) / a .
$$

Therefore $\Delta\left(M_{j}^{+} ; \mathcal{T}_{j} \cup\{T\}\right)$ has an $M_{0}$-minor, contrary to hypothesis. Thus we conclude that neither $\Delta\left(M_{1}^{+} ; \mathcal{T}_{1}\right)$ nor $\Delta\left(M_{2}^{+} ; \mathcal{T}_{2}\right)$ has an $M_{0}$-minor.

Let $M^{+}=M_{1}^{+} \oplus_{3} M_{2}^{+}$. Note that $\mathcal{T}_{1} \cup \mathcal{T}_{2}=\mathcal{T}_{i}$. But

$$
\Delta\left(M_{1}^{+} ; \mathcal{T}_{1}\right) \oplus_{3} \Delta\left(M_{2}^{+} ; \mathcal{T}_{2}\right)=\Delta\left(M^{+} ; \mathcal{T}_{i}\right)
$$

by Proposition 2.16. Let $P=E\left(M^{+}\right)-E\left(M_{i}\right)$, so that every element in $P$ is parallel to an element in $E\left(M_{i}\right)$, and $M^{+} \backslash P=M_{i}$. The method we use to construct the decomposition tree means that no triangle in $\mathcal{T}_{i}$ contains an element of $P$. Repeated application of Proposition 2.2 implies that

$$
\Delta\left(M^{+} ; \mathcal{T}_{i}\right) \backslash P=\Delta\left(M^{+} \backslash P ; \mathcal{T}_{i}\right)=\Delta\left(M_{i} ; \mathcal{T}_{i}\right) .
$$

Therefore $\Delta\left(M^{+} ; \mathcal{T}_{i}\right)$ has an $M_{0}$-minor.

Since neither $\Delta\left(M_{1}^{+} ; \mathcal{T}_{1}\right)$ nor $\Delta\left(M_{2}^{+} ; \mathcal{T}_{2}\right)$ has an $M_{0}$-minor, by considering Equation (4) and applying Lemma 2.19 we see that $\Delta_{T}\left(\Delta\left(M_{j}^{+} ; \mathcal{T}_{j}\right)\right)$ has an $M_{0}$-minor for some $j \in\{1,2\}$. But

$$
\Delta_{T}\left(\Delta\left(M_{j}^{+} ; \mathcal{T}_{j}\right)\right)=\Delta\left(M_{j}^{+} ; \mathcal{T}_{j} \cup\{T\}\right),
$$

so we have a contradiction to our choice of $\left(M_{i}, \mathcal{T}_{i}\right)$. This completes the proof of the lemma.

Lemma 4.11. Suppose that $M_{0}$ is a 3-connected binary matroid such that $\left|E\left(M_{0}\right)\right| \geq 4$ and $M_{0}$ has no triangles. Suppose also that $M$ is a binary matroid such that $\operatorname{si}(M)$ is 3-connected, and that $\mathcal{T}$ is a set of pairwise disjoint triangles of $M$. Let $\Phi(M, \mathcal{T})$ be a decomposition tree of $M$. If there is a leaf of $\Phi(M, \mathcal{T})$ labeled $\left(M_{i}, \mathcal{T}_{i}\right)$ where $\Delta\left(M_{i} ; \mathcal{T}_{i}\right)$ has an $M_{0}$-minor, then $\Delta(M ; \mathcal{T})$ has an $M_{0}$-minor. 
Proof. The proof is by induction of the size of the decomposition tree. If $\Phi(M, \mathcal{T})$ has one node then the result is obvious. Therefore assume that $\left(M_{1}^{+}, \mathcal{T}_{1} \cup\{T\}\right)$ and $\left(M_{2}^{+}, \mathcal{T}_{2} \cup\{T\}\right)$ are the children of $(M, \mathcal{T})$. We can assume that $\left(M_{i}, \mathcal{T}_{i}\right)$ is a leaf in the decomposition tree $\Phi\left(M_{1}^{+}, \mathcal{T}_{1} \cup\{T\}\right)$. The inductive hypothesis tells us that $\Delta\left(M_{1}^{+} ; \mathcal{T}_{1} \cup\{T\}\right)$ has an $M_{0}$-minor. Let $M^{+}=M_{1}^{+} \oplus_{3} M_{2}^{+}$. Proposition 4.8 says that

$$
\Delta\left(M_{1}^{+} ; \mathcal{T}_{1}\right) \oplus_{3} \Delta\left(M_{2}^{+} ; \mathcal{T}_{2}\right)=\Delta\left(M^{+} ; \mathcal{T}\right) .
$$

Suppose that $T_{1}, \ldots, T_{p}$ are the triangles in $\mathcal{T}_{2}$. We know from Proposition 2.14 that $\operatorname{si}\left(M_{2}^{+}\right)$is 3 -connected. Let $N^{(0)}=M_{2}^{+}$, and for $i=1, \ldots, p$ let $N^{(i)}$ be $\Delta_{T_{i}}\left(N^{(i-1)}\right)$. Thus $N^{(p)}=\Delta\left(M_{2}^{+} ; \mathcal{T}_{2}\right)$. If $\operatorname{si}\left(\Delta\left(M_{2}^{+} ; \mathcal{T}_{2}\right)\right)$ is not 3 -connected then there is an integer $i \in\{1, \ldots, p\}$ such that $\operatorname{si}\left(N^{(i-1)}\right)$ is 3 -connected but $\operatorname{si}\left(N^{(i)}\right)$ is not. Then there must be a $k$-separation $\left(X_{1}, X_{2}\right)$ of $N^{(i)}$ where $k<3$ and $r_{N^{(i)}}\left(X_{j}\right) \geq k$ for $j=1,2$. We will assume without loss of generality that $X_{1}$ contains at least two elements of $T_{i}$. Then $\left(X_{1} \cup T_{i}, X_{2}-T_{i}\right)$ is a $k^{\prime}$-separation of $N^{(i)}$ with the property that $k^{\prime} \leq k$, and $r_{N^{(i)}}\left(X_{1} \cup T_{i}\right), r_{N^{(i)}}\left(X_{2}-T_{i}\right) \geq k^{\prime}$. Hence we can assume that $T_{i}$ is contained in $X_{1}$. Now $r\left(N^{(i-1)}\right)=r\left(N^{(i)}\right)-1$ by Proposition 2.10. It follows from Proposition 2.2 that $\Delta_{T}\left(N^{(i-1)} \mid X_{1}\right)=N^{(i)} \mid X_{1}$. Therefore $r_{N^{(i-1)}}\left(X_{1}\right)=r_{N^{(i)}}\left(X_{1}\right)-1$ by Proposition 2.10. Now we see that $\left(X_{1}, X_{2}\right)$ is a $k$-separation of $N^{(i-1)}$ for some $k<3$. Moreover $r_{N^{(i-1)}}\left(X_{2}\right) \geq k$, and $r_{N^{(i-1)}}\left(X_{1}\right) \geq 2$ as $X_{1}$ contains a triangle of $N^{(i-1)}$. This contradicts the fact that $\operatorname{si}\left(N^{(i-1)}\right)$ is 3 -connected. We conclude that $\operatorname{si}\left(\Delta\left(M_{2}^{+} ; \mathcal{T}_{2}\right)\right)$ is 3-connected.

Since $M_{2}^{+}$has at least four rank-one flats (by construction), it follows easily that $\Delta\left(M_{2}^{+} ; \mathcal{T}_{2}\right)$ has at least four rank-one flats. Moreover $\Delta\left(M_{2}^{+} ; \mathcal{T}_{2}\right)$ contains a triangle, so $r\left(\operatorname{si}\left(\Delta\left(M_{2}^{+} ; \mathcal{T}_{2}\right)\right)\right) \geq 3$. No triangle can contain a cocircuit in a 3 -connected matroid. Therefore $T$ is a coindependent triangle in $\operatorname{si}\left(\Delta\left(M_{2}^{+} ; \mathcal{T}_{2}\right)\right)$, so the corank of $\operatorname{si}\left(\Delta\left(M_{2}^{+} ; \mathcal{T}_{2}\right)\right)$ is at least three. It follows without difficulty from Lemma 2.1 that $\Delta\left(M_{2}^{+} ; \mathcal{T}_{2}\right)$ has a minor $M^{\prime}$ such that $M^{\prime} \cong M\left(K_{4}\right)$ and $T$ is a triangle of $M^{\prime}$.

By Proposition 2.2 we see that $\Delta\left(M_{1}^{+} ; \mathcal{T}_{1}\right) \oplus_{3} \Delta\left(M_{2}^{+} ; \mathcal{T}_{2}\right)$ has $\left(\Delta\left(M_{1}^{+} ; \mathcal{T}_{1}\right)\right) \triangle\left(M^{\prime}\right)$ as a minor. But this last matroid is precisely $\Delta\left(M_{1}^{+} ; \mathcal{T}_{1} \cup\{T\}\right)$, which we know to have an $M_{0}$-minor. By considering Equation $(5)$ we conclude that $\Delta\left(M^{+} ; \mathcal{T}\right)$ has an $M_{0}$-minor. Since $\operatorname{si}\left(M^{+}\right) \cong M$ repeated application of Proposition 4.9 tells us that $\Delta(M ; \mathcal{T})$ has an $M_{0}$-minor, as desired.

Corollary 4.12. Suppose that $M_{0}$ is a 3-connected binary matroid such that $\left|E\left(M_{0}\right)\right| \geq 4$ and $M_{0}$ has no triangles. Suppose also that $M$ is a binary matroid such that $\operatorname{si}(M)$ is 3-connected. Let $\Phi(M, \varnothing)$ be a decomposition tree of $M$ and suppose that there is a leaf of $\Phi(M, \varnothing)$ labeled $\left(M_{i}, \mathcal{T}_{i}\right)$ where $\Delta\left(M_{i} ; \mathcal{T}_{i}\right)$ has an $M_{0}$-minor. Then $M$ has an $M_{0}$-minor. 
The following lemma (which follows easily from the previous results in this section), should make clear our strategy for deciding whether a binary matroid contains a minor from a particular class.

Lemma 4.13. Suppose that $\mathcal{M}$ is a collection of internally 4-connected binary matroids such that each matroid in $\mathcal{M}$ has at least four elements, and no matroid in $\mathcal{M}$ contains both a triangle and a triad. Let $\mathcal{M}_{\Upsilon}$ be the set of matroids in $\mathcal{M}$ that contain at least one triad. Suppose that $M$ is a 3-connected binary matroid and that there is a decomposition tree of $M$, the leaves of which are labeled $\left(M_{1}, \mathcal{T}_{1}\right), \ldots,\left(M_{p}, \mathcal{T}_{p}\right)$. Then $M$ has a minor in $\mathcal{M}$ if and only if:

(i) there is a leaf $\left(M_{i}, \mathcal{T}_{i}\right)$ such that $M_{i}$ has a minor in $\mathcal{M}$; or,

(ii) there is a leaf $\left(M_{i}, \mathcal{T}_{i}\right)$ such that $\Delta\left(M_{i} ; \mathcal{T}_{i}\right)$ has a minor in $\mathcal{M}_{\Upsilon}$.

(Indeed Lemma 4.13 is true even if we replace $\mathcal{M}$ in statement (i) of the lemma with $\mathcal{M}-\mathcal{M}_{\Upsilon}$.) In our case $\mathcal{M}$ is a subset of $\left\{M\left(K_{3,3}\right), M\left(K_{5}\right)\right.$, $\left.M^{*}\left(K_{3,3}\right), M^{*}\left(K_{5}\right)\right\}$ and either $M\left(K_{3,3}\right)$ or $M^{*}\left(K_{3,3}\right)$ is contained in $\mathcal{M}$. There are two tasks left to consider: For each leaf $\left(M_{i}, \mathcal{T}_{i}\right)$ of a decomposition tree we must decide whether $\operatorname{si}\left(M_{i}\right)$ has a minor in $\mathcal{M}$, and if this is not the case for any leaf we must decide whether $\Delta\left(M_{i} ; \mathcal{T}_{i}\right)$ has a minor in $\mathcal{M}_{\Upsilon}$.

The next result was first proved by Tutte [22] (see also [1, Section 7.2]).

Proposition 4.14. There is a polynomial-time algorithm which, given a binary matroid $M$, will either return a graph $G$ such that $M(G)=M$, or decide that no such graph exists.

It follows immediately that we can also decide in polynomial time whether a binary matroid is cographic or planar graphic.

Proposition 4.15. There is a polynomial-time algorithm which, given a binary matroid $M$, will decide whether $M$ is isomorphic to $\Delta_{r}$ for some integer $r \geq 3$, or to $\Upsilon_{r}$ for some even integer $r \geq 4$.

Proof. We claim that it is possible to decide in polynomial time whether a graph is isomorphic to a Möbius ladder. To see this, we observe that the rim edges of a Möbius ladder are precisely those edges in a unique cycle of length four. Thus we can identify the rim edges in polynomial time and verify that they form a Hamiltonian cycle $v_{0}, \ldots, v_{t}$. Let $n=\lfloor t / 2\rfloor$. If $t$ is even and each remaining edge joins a vertex $v_{i}$ to $v_{i+n}$, then the graph is a cubic Möbius ladder. If $t$ is odd and each remaining edge joins a vertex $v_{i}$ to either $v_{i+n}$ or $v_{i+n+1}$, then the graph is a quartic Möbius ladder. This completes the proof of the claim.

If a binary matroid $M$ is isomorphic to $\Delta_{r}$ then there is an element $e \in E(M)$ such that $M \backslash e$ is isomorphic to $M^{*}\left(C M_{2 r-2}\right)$, the bond matroid of the cubic Möbius ladder $C M_{2 r-2}$. Thus to decide whether $M \cong \Delta_{r}$, where $r=r(M)$, we consider each single-element deletion of $M$ in turn and decide in polynomial time whether it is isomorphic to $M^{*}\left(C M_{2 r-2}\right)$, using the algorithm of Proposition 4.14 and the claim in the previous paragraph. 
(Note that if $G$ is a graph such that $M^{*}(G)=M^{*}\left(C M_{2 r-2}\right)$ then $G=$ $C M_{2 r-2}$ by Whitney's 2-isomorphism theorem.) If $M \backslash e \cong M^{*}\left(C M_{2 r-2}\right)$ then $M \cong \Delta_{r}$ if and only if $e$ is in a circuit with all the spoke elements of the Möbius ladder.

If $M \cong \Upsilon_{r}$ then there is an element $e \in(M)$ such that $M \backslash e \cong$ $M^{*}\left(Q M_{r-1}\right)$, and $e$ is in a circuit with the spoke elements of the quartic Möbius ladder. Thus a similar argument works in the case of the triadic Möbius matroids.

Next we consider the problem of identifying when $\Delta\left(M_{i} ; \mathcal{T}_{i}\right)$ has a minor in $\mathcal{M}_{\Upsilon}$, where $\operatorname{si}\left(M_{i}\right)$ is an internally 4-connected matroid such that $\operatorname{si}\left(M_{i}\right) \in$ $\mathcal{E} \mathcal{X}(\mathcal{M})$ and $\mathcal{T}_{i}$ is a set of pairwise disjoint triangles of $M_{i}$.

Proposition 4.16. Suppose that $M$ is a binary matroid such that $r(M) \geq 3$ and $\operatorname{si}(M)$ is 3-connected. Let $\mathcal{T}$ be a set of pairwise disjoint triangles of $M$. If there are triangles $T_{1}, T_{2} \in \mathcal{T}$ such that $r_{M}\left(T_{1} \cup T_{2}\right)=2$ then $\Delta(M ; \mathcal{T})$ has an $M\left(K_{3,3}\right)$-minor.

Proof. Recall that we take the ground set of $\operatorname{si}(M)$ to be the set of rank-one flats of $M$. Let $T=\operatorname{cl}_{M}\left(T_{1}\right)$, so that $T$ is a triangle of $\operatorname{si}(M)$. By the hypotheses we know that $r(\operatorname{si}(M)) \geq 3$. Furthermore $\operatorname{si}(M)$ is 3-connected, so $T$ must be coindependent in $\operatorname{si}(M)$. Therefore $r^{*}(\operatorname{si}(M)) \geq 3$. Now Lemma 2.1 says that $\operatorname{si}(M)$ has a minor isomorphic to $M\left(K_{4}\right)$ in which $T$ is a triangle. Therefore $M$ has a minor $M^{\prime}$ isomorphic to the matroid produced from $M\left(K_{4}\right)$ by adding parallel elements to the points in a triangle; and moreover $T_{1}$ and $T_{2}$ are triangles of $M^{\prime}$.

It follows from Proposition 2.2 that $\Delta_{T_{2}}\left(\Delta_{T_{1}}\left(M^{\prime}\right)\right)$ is a minor of $\Delta_{T_{2}}\left(\Delta_{T_{1}}(M)\right)$. However $\Delta_{T_{2}}\left(\Delta_{T_{1}}\left(M^{\prime}\right)\right)$ is isomorphic to $M\left(K_{3,3}\right)$. Suppose that the members of $\mathcal{T}$ are $T_{1}, \ldots, T_{p}$. Let $M^{(0)}=M$, and for $i \in\{1, \ldots, p\}$, let $M^{(i)}$ be $\Delta_{T_{i}}\left(M^{(i-1)}\right)$. Thus $M^{(2)}$ has an $M\left(K_{3,3}\right)$-minor. If $\Delta(M ; \mathcal{T})$ does not have an $M\left(K_{3,3}\right)$-minor then there is an integer $i \in\{3, \ldots, p\}$ such that $M^{(i)}$ has no $M\left(K_{3,3}\right)$-minor, but $M^{(i-1)}$ does. Since $T_{i}$ is a triangle of $M^{(i-1)}$ and $M\left(K_{3,3}\right)$ has no triangles there is an element $a \in T_{i}$ such that $M^{(i-1)} \backslash a$ has an $M\left(K_{3,3}\right)$-minor. Proposition 2.9 implies that $M^{(i)}$ has an $M\left(K_{3,3}\right)$-minor. This contradiction completes the proof.

Proposition 4.17. Suppose that $M$ is a cographic matroid such that $r(M) \geq 3$ and $\operatorname{si}(M)$ is internally 4-connected. Let $\mathcal{T}$ be a set of pairwise disjoint triangles of $M$ and suppose that $r_{M}\left(T_{1} \cup T_{2}\right)>2$ for every pair of distinct triangles $T_{1}, T_{2} \in \mathcal{T}$. Then $\Delta(M ; \mathcal{T})$ is cographic, and if $M$ is planar graphic then so is $\Delta(M ; \mathcal{T})$.

Proof. Let $G$ be a graph such that $M=M^{*}(G)$. We can assume that $G$ contains no isolated vertices, and that furthermore, if $e$ is a loop of $M$, then there is a connected component of $G$ that contains only the single edge, $e$. Since $M$ does not consist solely of loops there is a connected component $G_{0}$ of $G$ such that $G_{0}$ contains more than one edge. Our assumption means 
that the minimum degree of $G_{0}$ is at least two. We shall say that a vertex of degree at least three in $G_{0}$ is a branch vertex and that a path between two distinct branch vertices is a branch. Thus a branch (along with all single-edge components of $G$ ) is a rank-one flat of $M$.

Suppose that $T$ is a triangle of $M$. Then $T$ is a minimal edge cut-set of $G$. Let $T=\left\{e_{1}, e_{2}, e_{3}\right\}$. Note that no branch of $G$ can contain more than one element of $T$. For $i=1,2,3$ let $l_{i}$ be the branch of $G$ that contains $e_{i}$, and let $L=\left\{l_{1}, l_{2}, l_{3}\right\}$. Then $G_{0} \backslash L$ contains exactly two connected components. Let $A$ and $B$ be the edge sets of these two components.

Assume that both $A$ and $B$ are non-empty. We initially suppose that both $A$ and $B$ contain at least two branches. If $A$ contains more than two branches then $\left(A \cup l_{1}, B \cup\left\{l_{2}, l_{3}\right\}\right)$ is a 3 -separation of $M$ that contradicts the fact that $\operatorname{si}(M)$ is internally 4 -connected. Therefore $A$ (and by symmetry, $B$ ) contains exactly two branches. Thus $\operatorname{si}(M)$ contains exactly seven elements. As $\operatorname{si}(M)$ is cographic this means that $r(\operatorname{si}(M))>3$. Now $\operatorname{si}(M)$ is internally 4-connected, and therefore contains no series pairs or coloops. Therefore $(\operatorname{si}(M))^{*}$ is a simple graphic matroid with seven elements and rank at most three, a contradiction.

This means that without loss of generality we can assume that $A$ contains precisely one branch. But if $v$ is the end vertex of $l_{1}$ that is contained in the subgraph induced by $A$, then $v$ must be incident with at least two branches in $A$, so we have a contradiction. Therefore we can assume that $A$ is empty. As $G_{0}$ contains no vertices of degree one it follows that there is a degree-three vertex $v_{T}$ that is incident with $l_{1}, l_{2}$, and $l_{3}$.

Suppose that the edge $f$ is contained in $l_{1}$ but is distinct from $e_{1}$. If we swap the labels on $e_{1}$ and $f$ then the bond matroid of the resulting graph is $M$. If $T_{1}$ and $T_{2}$ are distinct triangles in $\mathcal{T}$ then $v_{T_{1}} \neq v_{T_{2}}$ as $r_{M}\left(T_{1}, T_{2}\right)>2$. Therefore we can assume that every triangle in $\mathcal{T}$ consists of three edges incident with a degree-three vertex. It is clear that if we obtain $G^{\prime}$ from $G$ by replacing each member of $\mathcal{T}$ with a triangle then $M^{*}\left(G^{\prime}\right) \cong \Delta(M ; \mathcal{T})$. Moreover if $G$ is a planar graph then so is $G^{\prime}$.

Recall from Section 2.5 that the ground set of $\Delta_{r}$, the rank-r triangular Möbius matroid, is $\left\{e_{1}, \ldots, e_{r}, a_{1}, \ldots, a_{r-1}, b_{1}, \ldots, b_{r-1}\right\}$. For $i \in$ $\{1, \ldots, r-1\}$ the elements $e_{i}$ and $a_{i}$ are rim elements, while $b_{1}, \ldots, b_{r-1}$ are spoke elements and $e_{r}$ is the tip. The only triangles of $\Delta_{r}$ are sets of the form $\left\{a_{i}, e_{i}, e_{r}\right\}$ for $1 \leq i \leq r-1$, the sets $\left\{a_{i}, a_{i+1}, b_{i}\right\}$ and $\left\{e_{i}, e_{i+1}, b_{i}\right\}$ for $1 \leq i \leq r-2$, and the sets $\left\{a_{1}, e_{r-1}, b_{r-1}\right\}$ and $\left\{a_{r-1}, e_{1}, b_{r-1}\right\}$.

Lemma 4.18. Suppose that $M$ is a binary matroid such that $\operatorname{si}(M)=\Delta_{r}$ for some $r \geq 3$. Let $\mathcal{T}$ be a collection of pairwise disjoint triangles of $M$ such that $r_{M}\left(T_{1} \cup T_{2}\right)>2$ for all pairs of distinct triangles $T_{1}, T_{2} \in \mathcal{T}$. Then $\Delta(M ; \mathcal{T})$ has an $M\left(K_{3,3}\right)$-minor if and only if:

(i) There is a triangle $T \in \mathcal{T}$ such that $\operatorname{cl}_{M}(T)=\left\{a_{i}, e_{i}, e_{r}\right\}$ for some $1 \leq i \leq r-1$; 
(ii) There are triangles $T_{1}, T_{2} \in \mathcal{T}$ such that $\mathrm{cl}_{M}\left(T_{1}\right)=\left\{a_{i}, a_{i+1}, b_{i}\right\}$ and $\operatorname{cl}_{M}\left(T_{2}\right)=\left\{e_{i}, e_{i+1}, b_{i}\right\}$ for some $1 \leq i \leq r-2$; or,

(iii) There are triangles $T_{1}, T_{2} \in \mathcal{T}$ such that $\mathrm{cl}_{M}\left(T_{1}\right)=\left\{a_{1}, e_{r-1}, b_{r-1}\right\}$ and $\operatorname{cl}_{M}\left(T_{2}\right)=\left\{a_{r-1}, e_{1}, b_{r-1}\right\}$.

Proof. Remember that the ground set of $\operatorname{si}(M)$ is the set of rank-one flats of $M$. We can assume that $M$ has no loops. Suppose that there is a triangle $T \in \mathcal{T}$ such that $\operatorname{cl}_{M}(T)=\left\{a_{i}, e_{i}, e_{r}\right\}$. Let $P$ be a set of elements such that $M \backslash P \cong \operatorname{si}(M)$, so that $M \backslash P=\Delta_{r}$. (We are abusing notation here: For example, $e_{r}$ is both an element of the ground set of $M \backslash P$, and a rank-one flat of $M$.) We can assume that $T$ is a triangle of $M \backslash P$. Now Claim 4.6 of [12] implies that $\Delta_{T}(M \backslash P)$ has an $M\left(K_{3,3}\right)$-minor. Therefore $\Delta_{T}(M) \backslash P$ has an $M\left(K_{3,3}\right)$-minor, and so does $\Delta_{T}(M)$. Suppose that the members of $\mathcal{T}$ are $T_{1}, \ldots, T_{p}$. Proposition 2.8 implies that we can assume $T=T_{1}$. Let $M^{(0)}=M$, and for $i \in\{1, \ldots, p\}$ let $M^{(i)}=\Delta_{T_{i}}\left(M^{(i-1)}\right)$. If $\Delta(M ; \mathcal{T})$ does not have an $M\left(K_{3,3}\right)$-minor then there must be an integer $i$ such that $M^{(i)}$ does not have an $M\left(K_{3,3}\right)$-minor, but $M^{(i-1)}$ does. Now we can obtain a contradiction exactly as in the proof of Proposition 4.16.

Next we will assume that $T_{1}$ and $T_{2}$ are members of $\mathcal{T}$ such that $\operatorname{cl}_{M}\left(T_{1}\right)=\left\{a_{i}, a_{i+1}, b_{i}\right\}$ and $\operatorname{cl}_{M}\left(T_{2}\right)=\left\{e_{i}, e_{i+1}, b_{i}\right\}$. Let $P$ be a set of elements such that $M \backslash P=\Delta_{r}$. We can assume that $T_{1}$ is a triangle of $M \backslash P$. Furthermore there is an element $b \in P$ such that $\operatorname{cl}_{M}(\{b\})=b_{i}$. We can assume that $T_{2}=\left\{e_{i}, e_{i+1}, b\right\}$ and that $T_{2}$ is a triangle of $M \backslash(P-b)$. Claim 4.7 of [12] tells us that $\Delta_{T_{2}}\left(\Delta_{T_{1}}(M \backslash(P-b))\right)$ has an $M\left(K_{3,3}\right)$-minor, so $\Delta_{T_{2}}\left(\Delta_{T_{1}}(M)\right)$ has an $M\left(K_{3,3}\right)$-minor. Exactly as before we can show that $\Delta(M ; \mathcal{T})$ has an $M\left(K_{3,3}\right)$-minor. The same argument shows that $\Delta(M ; \mathcal{T})$ has an $M\left(K_{3,3}\right)$-minor if there are triangles $T_{1}, T_{2} \in \mathcal{T}$ such that $\operatorname{cl}_{M}\left(T_{1}\right)=\left\{a_{1}, e_{r-1}, b_{r-1}\right\}$ and $\operatorname{cl}_{M}\left(T_{2}\right)=\left\{a_{r-1}, e_{1}, b_{r-1}\right\}$.

This completes the "if" direction of the proof. To prove the "only if" direction we need to introduce a family of matroids derived from the triangular Möbius matroids. For any positive integer $r$ let $\Delta_{r}^{\circ}$ be obtained from $\Delta_{r}$ by adding $a_{i}^{\prime}$ in parallel to $a_{i}$ and $e_{i}^{\prime}$ in parallel to $e_{i}$, for $1 \leq i \leq r-1$.

Claim 4.19. Suppose that $N$ is a restriction of $\Delta_{r}^{\circ}$ for some $r \geq 3$ and that $\mathcal{T}$ is a set of pairwise disjoint triangles of $M$ with the property that $r_{M}\left(T_{1} \cup T_{2}\right)>2$ for every pair of distinct triangles $T_{1}, T_{2} \in \mathcal{T}$, and $\mathcal{T}$ does not satisfy conditions (i), (ii), or (iii) of Lemma 4.18. Then $\Delta(N ; \mathcal{T})$ is a restriction of $\Delta_{s}^{\circ}$ for some $s \geq 3$.

Proof. Let $T_{1}, \ldots, T_{p}$ be the members of $\mathcal{T}$. The proof is by induction on $p$. If $\mathcal{T}$ is empty then the result is trivial, so we will assume that $p \geq 1$. Note that $T_{1}$ contains $b_{i}$ for some $i \in\{1, \ldots, r-1\}$, and that no other member of $\mathcal{T}$ contains $b_{i}$. Lemma 4.8 of [12] says that $\Delta_{T_{1}}\left(\Delta_{r}^{\circ}\right)$ is isomorphic to a restriction of $\Delta_{r+1}^{\circ}$, and this isomorphism takes spoke elements other than $b_{i}$ to spoke elements. Since $N$ is a restriction of $\Delta_{r}^{\circ}$ it follows from Proposition 2.2 that $\Delta_{T_{1}}(N)$ is isomorphic to a restriction of $\Delta_{r+1}^{\circ}$, where 
the isomorphism again takes spoke elements other than $b_{i}$ to spoke elements. Now it is easy to see that if we apply this isomorphism to $\left\{T_{2}, \ldots, T_{p}\right\}$ we obtain a collection of pairwise disjoint triangles of $\Delta_{r+1}^{\circ}$ that does not satisfy condition (i), (ii), or (iii) of the lemma. The claim now follows by induction.

We return to the proof of Lemma 4.18. Note that $\Delta_{r}^{\circ}$ can be obtained from $M$ by possibly adding and deleting parallel elements. Moreover, if $\mathcal{T}$ is a collection of pairwise disjoint triangles of $M$ such that $\mathcal{T}$ does not satisfy condition (i), (ii), or (iii) of the lemma, then $\mathcal{T}$ is also a collection of pairwise disjoint triangles of $\Delta_{r}^{\circ}$. Since $\Delta_{r}$, and hence $\Delta_{r}^{\circ}$ does not have an $M\left(K_{3,3}\right)$-minor for any $r \geq 3$ it follows from Claim 4.19 that $\Delta\left(\Delta_{r}^{\circ} ; \mathcal{T}\right)$ does not have an $M\left(K_{3,3}\right)$-minor. Propositions 2.2 and 4.9 imply that $\Delta(M ; \mathcal{T})$ has no $M\left(K_{3,3}\right)$-minor, and this completes the proof.

Now we prove our main result.

Theorem 1.2. Suppose that $\mathcal{M}$ is a subset of the family $\left\{M\left(K_{3,3}\right), M\left(K_{5}\right)\right.$, $\left.M^{*}\left(K_{3,3}\right), M^{*}\left(K_{5}\right)\right\}$ such that $\mathcal{M}$ contains either $M\left(K_{3,3}\right)$ or $M^{*}\left(K_{3,3}\right)$. There is an algorithm which, given a matrix $A$ over $\mathrm{GF}(2)$ with $n$ columns (and at most $n$ rows), will decide whether $M[A]$ has a minor in $\mathcal{M}$, in time that is bounded by a polynomial function of $n$.

Proof. Clearly if we can decide membership in a class of matroids in polynomial time, then we can also decide membership in the dual class. Therefore we will always assume that $M\left(K_{3,3}\right) \in \mathcal{M}$. It is not difficult to see that it will suffice to construct a polynomial-time algorithm which will decide membership in $\mathcal{E} \mathcal{X}(\mathcal{M})$ when $M[A]$ is 3-connected.

Let $M=M[A]$. By Proposition 4.5 we can construct a decomposition tree $\Phi(M, \varnothing)$ in polynomial-time. First let us assume that $M^{*}\left(K_{5}\right) \notin \mathcal{M}$. Note that if $\left(M_{i}, \mathcal{T}_{i}\right)$ is a leaf of $\Phi(M, \varnothing)$ then $\left|E\left(\operatorname{si}\left(M_{i}\right)\right)\right| \leq n$. By Propositions 4.14 and 4.15 we can decide in polynomial time whether $\operatorname{si}\left(M_{i}\right)$ is cographic, planar graphic, or isomorphic to a Möbius matroid, for each leaf $\left(M_{i}, \mathcal{T}_{i}\right)$ of $\Phi(M, \varnothing)$. Certainly we can decide whether $\operatorname{si}\left(M_{i}\right)$ is isomorphic to one of a finite number of fixed matroids. Therefore, by examining Theorems $1.1,3.2,3.5$, and 3.7 , we see that we can decide in polynomial time whether $M_{i}$ has a minor in $\mathcal{M}$, for each leaf $\left(M_{i}, \mathcal{T}_{i}\right)$. If some $M_{i}$ has a minor in $\mathcal{M}$, then so does $M$, by Proposition 4.6, in which case we can stop. Therefore we suppose that each $M_{i}$ belongs to $\mathcal{E} \mathcal{X}(\mathcal{M})$.

Since the only matroid in $\mathcal{M}$ with any triads is $M\left(K_{3,3}\right)$, it follows from Proposition 4.7 that if $M$ has a minor in $\mathcal{M}$ then $M$ must have an $M\left(K_{3,3}\right)$-minor. By Lemma 4.10 and Corollary 4.12 this is true if and only if there is a leaf $\left(M_{i}, \mathcal{T}_{i}\right)$ such that $\Delta\left(M_{i} ; \mathcal{T}_{i}\right)$ has an $M\left(K_{3,3}\right)$-minor. Note that by definition of 3 -sum it follows that $r(\operatorname{si}(M)) \geq 3$ for every leaf $\left(M_{i}, \mathcal{T}_{i}\right)$. Propositions 4.16 and 4.17 imply that if $\operatorname{si}(M)$ is cographic or planar graphic then $\Delta\left(M_{i} ; \mathcal{T}_{i}\right)$ has an $M\left(K_{3,3}\right)$-minor if and only there is a pair of distinct triangles in $\mathcal{T}_{i}$ whose union has rank two. Certainly we can 
decide whether this is true in polynomial time. Note that the triadic Möbius matroids have no triangles. If $\operatorname{si}\left(M_{i}\right)$ is a triangular Möbius matroid then we can decide whether $\Delta\left(M_{i} ; \mathcal{T}_{i}\right)$ has an $M\left(K_{3,3}\right)$-minor by examining $\mathcal{T}_{i}$ and using Proposition 4.16 and Lemma 4.18. Suppose that $\operatorname{si}(M)$ is isomorphic to one of a finite number of fixed matroids. If there are two distinct triangles in $\mathcal{T}_{i}$ whose union has rank two then $\Delta\left(M_{i} ; \mathcal{T}_{i}\right)$ has an $M\left(K_{3,3}\right)$-minor. Therefore we will assume that this is not the case. It follows that $\left|\mathcal{T}_{i}\right|$ is bounded by some fixed constant. Now we can obtain the matroid $M^{\prime}$ by adding parallel elements to $\operatorname{si}\left(M_{i}\right)$ in such a way that there is a set $\mathcal{T}$ of pairwise disjoint triangles in $M^{\prime}$, such that there is a natural bijection between triangles in $\mathcal{T}$ and triangles in $\mathcal{T}_{i}$. Moreover $\left|E\left(M^{\prime}\right)\right|$ is bounded by some constant. Therefore we can decide in constant time whether $\Delta\left(M^{\prime} ; \mathcal{T}\right)$ has an $M\left(K_{3,3}\right)$-minor, and since $M_{i}$ is obtained from $M^{\prime}$ by possibly adding parallel elements it follows from Propositions 2.2 and 4.9 that $\Delta\left(M^{\prime} ; \mathcal{T}\right)$ has an $M\left(K_{3,3}\right)$-minor if and only if $\Delta\left(M_{i} ; \mathcal{T}_{i}\right)$ does.

This completes the proof of the theorem in the case that $\mathcal{M}$ does not contain $M^{*}\left(K_{5}\right)$. If $M^{*}\left(K_{5}\right) \in \mathcal{M}$ then we proceed as before and construct a decomposition tree $\Phi(M, \varnothing)$. By examining Theorem 3.4, Theorem 3.6, and Theorem 3.8, we see that to decide whether $M_{i} \in \mathcal{E} \mathcal{X}(\mathcal{M})$ for each leaf $\left(M_{i}, \mathcal{T}_{i}\right)$ we need only decide whether $\operatorname{si}\left(M_{i}\right)$ is planar graphic or isomorphic to one of a finite set of sporadic matroids. This can clearly be done in polynomial time. Once again we will assume that $M_{i} \in \mathcal{E} \mathcal{X}(\mathcal{M})$ for each leaf $\left(M_{i}, \mathcal{T}_{i}\right)$, for otherwise we are done. Now Lemma 4.10 and Corollary 4.12 imply that $M$ has a minor in $\mathcal{M}$ if and only if there is some leaf $\left(M_{i}, \mathcal{T}_{i}\right)$ such that $\Delta\left(M_{i} ; \mathcal{T}_{i}\right)$ has either an $M\left(K_{3,3}\right)$-minor, or an $M^{*}\left(K_{5}\right)$-minor. We can decide whether this is true in constant time if $\operatorname{si}\left(M_{i}\right)$ is isomorphic to one of a set of sporadic matroids. Therefore the only thing left to do is decide whether $\Delta\left(M_{i} ; \mathcal{T}_{i}\right)$ has a minor isomorphic to $M\left(K_{3,3}\right)$ or $M^{*}\left(K_{5}\right)$ in the case that $\operatorname{si}\left(M_{i}\right)$ (and hence $M_{i}$ ) is planar graphic.

If there are distinct triangles $T_{1}, T_{2} \in \mathcal{T}_{i}$ such that $r_{M_{i}}\left(T_{1} \cup T_{2}\right)=2$ then $\Delta\left(M_{i} ; \mathcal{T}_{i}\right)$ has an $M\left(K_{3,3}\right)$-minor by Proposition 4.16. If no two triangles in $\mathcal{T}$ satisfy this condition then $\Delta\left(M_{i} ; \mathcal{T}_{i}\right)$ is planar graphic by Proposition 4.17, and hence has no minor isomorphic to $M\left(K_{3,3}\right)$ or $M^{*}\left(K_{5}\right)$. This completes the proof of the theorem.

We conclude this section by noting that Möbius matroids have branchwidth four, so by using Hliněný's [7] matroid analogue of Courcelle's [3] theorem, the minor-testing algorithm for graphs, due to Robertson and Seymour [17], and Theorem 1.1, we can decide in polynomial-time whether a represented internally 4-connected binary matroid belongs to any class of the form $\mathcal{E} \mathcal{X}\left(\mathcal{N} \cup\left\{M\left(K_{3,3}\right)\right\}\right)$, where $\mathcal{N}$ is any set of binary matroids. However, any such algorithm relies upon (and in fact implies) a non-constructive result from the Graph Minors project of Robertson and Seymour. Therefore, this fact does not make Theorem 1.2 redundant, as that result is constructive, 
in the sense that the algorithms it describes could be implemented without too much difficulty.

4.2. Oracle algorithms. Up to this point we have assumed that all binary matroids are represented by matrices over $\mathrm{GF}(2)$. In this section we will assume that a matroid $M$ (not necessarily binary) is represented by an oracle. Suppose that $\mathcal{M}$ is a subcollection of the family $\left\{M\left(K_{3,3}\right), M\left(K_{5}\right)\right.$, $\left.M^{*}\left(K_{3,3}\right), M^{*}\left(K_{5}\right)\right\}$ and that $\mathcal{M}$ contains either $M\left(K_{3,3}\right)$ or $M^{*}\left(K_{3,3}\right)$. If we wish to decide whether $M$ has a minor in $\mathcal{M}$, then we can construct a partial representation $A$ of $M$, and run the algorithm of Theorem 1.2 upon the binary matroid $M\left[I_{r(M)} \mid A\right]$. If $M$ is a binary matroid then this algorithm will return the correct answer. However if $M$ is non-binary then we cannot be certain that this is the case. If the algorithm indicates that $M\left[I_{r(M)} \mid A\right]$ has a minor in $\mathcal{M}$ then all we can be sure of is that either $M$ has a minor in $\mathcal{M}$, or $M$ is non-binary. Similarly, if the algorithm decides that $M\left[I_{r(M)} \mid A\right]$ has no minor in $\mathcal{M}$ then either $M$ has no minor in $\mathcal{M}$, or $M$ is non-binary. Thus we can decide whether $M$ has a minor in $\mathcal{M} \cup\left\{U_{2,4}\right\}$, but we cannot decide which of these matroids it has as a minor.

In some sense this is the best we can hope for, as a famous example of Seymour's [19] shows: For $r \geq 3$ let $\left\{e_{1}, \ldots, e_{r}\right\}$ be the standard basis of the vector space over $\mathrm{GF}(2)$ with dimension $r$. Let $d$ be the sum of $e_{1}, \ldots, e_{r}$, and for $1 \leq i \leq r$ let $d_{i}$ be the sum of $d$ and $e_{i}$. Let $N_{r}$ be the binary matroid represented by the set $\left\{e_{1}, \ldots, e_{r}, d_{1}, \ldots, d_{r}\right\}$. If $H$ is a subset of $E\left(N_{r}\right)$ such that $\left|H \cap\left\{d_{1}, \ldots, d_{r}\right\}\right|$ is odd and $\left|H \cap\left\{e_{i}, d_{i}\right\}\right|=1$ for $1 \leq i \leq r$, then $H$ is a circuit-hyperplane of $N_{r}$. Let $N_{r}(H)$ be the matroid obtained from $N_{r}$ by relaxing $H$. It is not difficult to prove by induction on $r$ that $N_{r}$ has no minor in the set $\left\{M\left(K_{3,3}\right), M\left(K_{5}\right), M^{*}\left(K_{3,3}\right), M^{*}\left(K_{5}\right)\right\}$. Moreover, $N_{r}(H)$ is non-binary. In the worst case, an oracle algorithm will have to check each of the $2^{r-1}$ candidate sets $H$ to decide whether the matroid it is considering is isomorphic to $N_{r}$, or $N_{r}(H)$. Therefore we have the following result.

Proposition 4.20. Let $\mathcal{M}$ be a subset of $\left\{M\left(K_{3,3}\right), M\left(K_{5}\right), M^{*}\left(K_{3,3}\right)\right.$, $\left.M^{*}\left(K_{5}\right)\right\}$. There is no polynomial function $p$ such that one can decide whether a matroid $M$ belongs to $\mathcal{E} \mathcal{X}(\mathcal{M})$ using at most $p(|E(M)|)$ calls to an oracle.

We note that the binary matroid $N_{r}$ contains many 3-separations, and is therefore far from being internally 4 -connected. If we restrict our attention to internally 4-connected matroids the situation changes dramatically. Seymour [19] shows that there is a polynomial $p$ and an algorithm which, given a matroid $M$ (not necessarily binary), will either output a graph $G$ such that $M=M(G)$, or decide that no such graph exists, using at most $p(|E(M)|)$ calls to an oracle. Using a similar strategy to that in the proof of Proposition 4.15 we can show that it is possible to decide whether a matroid $M$ is isomorphic to a Möbius matroid, using only a polynomial number of calls to an oracle. Since it is obviously possible to decide whether a matroid 
$M$ is isomorphic to one of a finite number of sporadic matroids using a constant number of oracle calls, the next proposition follows from the results of Section 3.

Proposition 4.21. Let $\mathcal{M}$ be a subset of $\left\{M\left(K_{3,3}\right), M\left(K_{5}\right), M^{*}\left(K_{3,3}\right)\right.$, $\left.M^{*}\left(K_{5}\right)\right\}$ that contains either $M\left(K_{3,3}\right)$ or $M^{*}\left(K_{3,3}\right)$. There is a polynomial function $p$ such that one can decide whether an internally 4-connected matroid $M$ belongs to $\mathcal{E} \mathcal{X}(\mathcal{M})$, using at most $p(|E(M)|)$ calls to an oracle.

5. Maximum-Sized BinaRY Matroids With No $M\left(K_{3,3}\right)$

Suppose that $\mathcal{M}$ is a family of matroids. We say that $M \in \mathcal{M}$ is a maximum-sized member of $\mathcal{M}$ if $M$ is simple, and whenever $M^{\prime} \in \mathcal{M}$ is a simple matroid with the same rank as $M$, then $\left|E\left(M^{\prime}\right)\right| \leq|E(M)|$.

Kung [10] investigated the maximum-sized members of $\mathcal{E} \mathcal{X}\left(M\left(K_{3,3}\right)\right)$, and showed that such a matroid $M$ satisfies $|E(M)| \leq 10 r(M)$. We complete this programme by characterizing the maximum-sized members of $\mathcal{E} \mathcal{X}\left(M\left(K_{3,3}\right)\right)$. As a consequence, we show that if $M$ is a rank-r maximum-sized member of $\mathcal{E} \mathcal{X}\left(M\left(K_{3,3}\right)\right)$, then $|E(M)|=14 r / 3-\alpha(r)$, where $\alpha(r)$ takes on the values $7,11 / 3$, and $19 / 3$ according to the residue of $r$ modulo 3 .

Suppose that $M \in \mathcal{E} \mathcal{X}\left(M\left(K_{3,3}\right)\right)$ and that $T$ is a triangle of $M$. We say that $T$ is an allowable triangle of $M$ if $\Delta_{T}(M)$ has no $M\left(K_{3,3}\right)$-minor.

Lemma 5.1. Suppose that $M$ is a 3-connected member of $\mathcal{E X}\left(M\left(K_{3,3}\right)\right)$. If either

(i) $M$ has an allowable triangle; or,

(ii) $r(M)>4$,

then $|E(M)| \leq 4 r(M)-5$.

Proof. Let $M$ be a 3-connected member of $\mathcal{E X}\left(M\left(K_{3,3}\right)\right)$. Let $r=r(M)$. Note that if $M$ satisfies statement (i) or (ii), then $r \geq 2$. Moreover, if $r=2$, then $|E(M)|=3$, so $|E(M)| \leq 4 r-5$. Henceforth we assume that $r \geq 3$. Suppose that $M$ is internally 4 -connected. Theorem 1.1 implies that $M$ is either cographic, isomorphic to a Möbius matroid, or isomorphic to one of the sporadic matroids in Theorem 1.1.

Suppose that $M$ is a sporadic matroid. There are no sporadic matroids with rank 3. If $r>4$ then it is easily confirmed that $|E(M)| \leq 4 r-5$. If $r=4$ and $M$ has an allowable triangle, then $|E(M)| \leq 11$ (see Appendix $\mathrm{C}$ of [12]), and therefore $|E(M)| \leq 4 r-5$.

Suppose that $M$ is a Möbius matroid. A rank- $r$ triangular Möbius matroid contains $3 r-2$ elements, and a rank- $r$ triadic Möbius matroid has $2 r-1$ elements. Since $r \geq 3$ it follows that $|E(M)| \leq 4 r-5$.

Now assume that $M$ is cographic. Suppose that $G$ is a graph such that $M=M^{*}(G)$. As $r \geq 3$, it follows that the minimum degree of $G$ is at least three, because $M$ is internally 4 -connected. By splitting vertices, we can see that $|E(M)|$ is no greater than the number of elements in a rank- $r$ cographic matroid corresponding to a graph that is regular with degree three. Such 
a cographic matroid contains exactly $3 r-3$ elements. It again follows that $|E(M)| \leq 4 r-5$.

Now we assume that the lemma is false, and that $M$ is a counterexample with the smallest possible rank. The previous paragraphs imply that $M$ is not internally 4-connected.

Let $\left(X_{1}, X_{2}\right)$ be an exact 3 -separation of $M$ such that $\left|X_{i}\right| \geq 4$ for $i=1,2$. By Proposition 2.12 there are binary matroids $M_{1}$ and $M_{2}$ on the ground sets $X_{1} \cup T$ and $X_{2} \cup T$ respectively such that $T \cap\left(X_{1} \cup X_{2}\right)=\varnothing$ and $M=M_{1} \oplus_{3} M_{2}$. Assume that the ranks of $M_{1}$ and $M_{2}$ are $r_{1}$ and $r_{2}$ respectively. Since the 3 -sum $M_{1} \oplus_{3} M_{2}$ is defined, it follows that $T$ does not contain a cocircuit in either $M_{1}$ or $M_{2}$. Thus $r_{i}=r_{M}\left(X_{i}\right)$ for $i=1,2$. Therefore $r_{1}+r_{2}-r=2$. Because $\left|E\left(M_{i}\right)\right| \geq 7$ and every parallel pair of $M_{i}$ involves a member of $T$ it follows that $r_{i}>2$ for $i=1,2$. Hence $r_{1}, r_{2}<r$. Proposition 2.14 says that $\operatorname{si}\left(M_{1}\right)$ and $\operatorname{si}\left(M_{2}\right)$ are 3 -connected. Moreover $M_{i}$ (and hence $\operatorname{si}\left(M_{i}\right)$ ) has no $M\left(K_{3,3}\right)$-minor for $i=1,2$ by Proposition 2.13 . Therefore we can apply the lemma to $\mathrm{si}\left(M_{1}\right)$ and $\mathrm{si}\left(M_{2}\right)$ by our inductive assumption.

If $T$ is not an allowable triangle of $M_{i}$ then $\Delta_{T}\left(M_{i}\right)$ has an $M\left(K_{3,3}\right)$-minor, and therefore $M$ has an $M\left(K_{3,3}\right)$-minor by Proposition 2.15, a contradiction. Therefore $T$ is an allowable triangle in both $M_{1}$ and $M_{2}$. For $i=1,2$ let $n_{i}=\left|E\left(\operatorname{si}\left(M_{i}\right)\right)\right|$. As the lemma holds for $\operatorname{si}\left(M_{1}\right)$ and $\operatorname{si}\left(M_{2}\right)$ we deduce that $n_{i} \leq 4 r_{i}-5$ for $i=1,2$.

The only parallel classes of $M_{i}$ have size two, and contain an element of $T$. Note that no element in $T$ can be in a parallel pair in both $M_{1}$ and $M_{2}$, for that would imply that $M=M_{1} \oplus_{3} M_{2}$ has a parallel pair. Let $m$ be the number of elements in $T$ that are in a parallel pair in either $M_{1}$ or $M_{2}$. Then

$$
\begin{aligned}
|E(M)|=n_{1} & +n_{2}-m-2(3-m)=n_{1}+n_{2}+m-6 \\
& \leq\left(4 r_{1}-5\right)+\left(4 r_{2}-5\right)-3=4\left(r_{1}+r_{2}-2\right)-5=4 r-5 .
\end{aligned}
$$

Therefore $M$ is not a counterexample to the lemma. This contradiction completes the proof.

For integers $r \geq 2$ we recursively define the classes of rank-r matroids $\mathcal{P}_{r}$ as follows:

$$
\mathcal{P}_{2}=\{\mathrm{PG}(1,2)\}, \quad \mathcal{P}_{3}=\{\mathrm{PG}(2,2)\}, \quad \mathcal{P}_{4}=\{\mathrm{PG}(3,2)\}
$$

and for $r>4$ we define $\mathcal{P}_{r}$ to be the set

$$
\left\{P(M, \mathrm{PG}(3,2)) \mid M \in \mathcal{P}_{r-3}\right\},
$$

where $P(M, \mathrm{PG}(3,2))$ is the parallel connection of $M$ and $\mathrm{PG}(3,2)$ (see $[15$, Section 7.1]) along an arbitrary basepoint. It is well known that parallel connections can be expressed as 2-sums. It follows that if $M_{0}$ is a 3-connected binary matroid, and neither $M_{1}$ nor $M_{2}$ has $M_{0}$ as a minor, the parallel connection of $M_{1}$ and $M_{2}$ does not have an $M_{0}$-minor ([12, Proposition 2.20]). The next result follows from this fact and induction. 
Proposition 5.2. Let $r \geq 2$ be an integer, and suppose that $M \in \mathcal{P}_{r}$. Then $M$ has no $M\left(K_{3,3}\right)$-minor.

Note that for each $r \geq 2$ the matroids in $\mathcal{P}_{r}$ have the same size. Let $f(r)$ denote this common size. The precise value of $f(r)$ depends on the residue class of $r$ modulo 3 , as shown in Table 1 .

\begin{tabular}{|c|c|c|}
\hline Rank $r$ & Size & $f(r)$ \\
\hline$r=3 k$ & $14 k-7$ & $14 r / 3-7$ \\
$r=3 k+1$ & $14 k+1$ & $14 r / 3-11 / 3$ \\
$r=3 k+2$ & $14 k+3$ & $14 r / 3-19 / 3$ \\
\hline
\end{tabular}

TABLE 1 . The size of matroids in $\mathcal{P}_{r}$.

Define $\alpha(r)=14 r / 3-f(r)$ so that each member of $\mathcal{P}_{r}$ has $14 r / 3-\alpha(r)$ elements and $\alpha(r)$ depends only on the value of $r$ modulo 3 .

Now we can prove the main result of this section.

Theorem 5.3. A matroid $M$ with rank $r \geq 2$ is a maximum-sized member of $\mathcal{E} \mathcal{X}\left(M\left(K_{3,3}\right)\right)$ if and only if $M \in \mathcal{P}_{r}$.

Proof. We prove the "only if" direction first. Assume that $M$ is a maximumsized member of $\mathcal{E} \mathcal{X}\left(M\left(K_{3,3}\right)\right)$ and that $M$ does not belong to $\mathcal{P}_{r}$ for any $r \geq 2$. Let $r=r(M)$ and assume that no such counterexample exists with rank less than $r$. The theorem is easily seen to be true for matroids of rank at most four, so $r>4$. Obviously $M$ is at least as large as a member of $\mathcal{P}_{r}$, so $|E(M)| \geq 14 r / 3-7$. As $r>4$ we see that $14 r / 3-7>4 r-5$, so $M$ is not 3 -connected by Lemma 5.1. Hence there is an exact $k$-separation $\left(X_{1}, X_{2}\right)$ of $M$, where $k<3$.

If $k=1$ then $M=M_{1} \oplus M_{2}$, where $M_{i}=M \mid X_{i}$ for $i=1$, 2. Neither $M_{1}$ nor $M_{2}$ has an $M\left(K_{3,3}\right)$-minor, and certainly both are simple. Suppose that $r_{i}=r\left(M_{i}\right)$ for $i=1,2$, so that $r=r_{1}+r_{2}$. Since $M$ is simple, $r_{i}>0$ and hence $r_{i}<r$ for $i=1,2$. Therefore we can apply our inductive hypothesis and conclude that $\left|E\left(M_{i}\right)\right| \leq 14 r_{i} / 3-\alpha\left(r_{i}\right)$ for $i=1,2$. Now

$$
|E(M)|=\left|E\left(M_{1}\right)\right|+\left|E\left(M_{2}\right)\right| \leq 14 r / 3-\left(\alpha\left(r_{1}\right)+\alpha\left(r_{2}\right)\right) .
$$

But $\alpha\left(r_{1}\right)+\alpha\left(r_{2}\right)>7$, regardless of the residue classes of $r_{1}$ and $r_{2}$ modulo 3 , so $|E(M)|<14 r / 3-7$, contradicting our earlier conclusion.

Now we can assume that $M$ is connected, and that $k=2$. Then Proposition 2.11 says that $M=M_{1} \oplus_{2} M_{2}$, where the ground set of $M_{i}$ is $X_{i} \cup p$ for $i=1,2$, and $p \notin X_{1} \cup X_{2}$. Let $r_{i}=r\left(M_{i}\right)$ for $i=1,2$. As $p$ is not a loop or coloop in $M_{1}$ or $M_{2}$ it follows from Proposition 2.3 that $r_{i}=r_{M}\left(X_{i}\right)$ for $i=1,2$. Thus $r_{1}+r_{2}-r=1$. As $M$ has no parallel pairs it follows that $r_{1}, r_{2} \geq 2$. Therefore $r_{1}, r_{2}<r$. Proposition 2.3 also implies that neither $M_{1}$ nor $M_{2}$ has an $M\left(K_{3,3}\right)$-minor.

The fact that $M$ has no parallel pairs means that for all $i \in\{1,2\}$, either $M_{i}$ is simple or $M_{i}$ contains no loops and exactly one parallel pair, which 
contains $p$. Suppose that both $M_{1}$ and $M_{2}$ are simple. Let $M_{1}^{\prime}$ be obtained by adding an element in parallel to $p$. Then $M_{1}^{\prime} \oplus_{2} M_{2}$ is simple, and has no $M\left(K_{3,3}\right)$-minor by [12, Proposition 2.20]. Moreover $M_{1}^{\prime} \oplus_{2} M_{2}$ has more elements than $M=M_{1} \oplus_{2} M_{2}$, a contradiction. Therefore either $M_{1}$ or $M_{2}$ contains a single parallel pair, and this pair contains $p$. It cannot be the case that both $M_{1}$ and $M_{2}$ contain a parallel pair, for then $M$ would have a parallel pair.

Suppose that $M_{1}$ is simple, and that $M^{\prime}$ is a simple rank- $r_{1}$ matroid in $\mathcal{E} \mathcal{X}\left(M\left(K_{3,3}\right)\right)$ such that $\left|E\left(M^{\prime}\right)\right|>\left|E\left(M_{1}\right)\right|$. Let us assume that $E\left(M^{\prime}\right) \cap$ $E\left(M_{2}\right)=\{p\}$. We can also assume that $p$ is not a coloop of $M^{\prime}$, as $M^{\prime}$ is not isomorphic to $U_{r_{1}, r_{1}}$. Therefore $M^{\prime} \oplus_{2} M_{2}$ is defined, and has no $M\left(K_{3,3}\right)$-minor by [12, Proposition 2.20]. Moreover $M^{\prime} \oplus_{2} M_{2}$ is simple and $\left|E\left(M^{\prime} \oplus_{2} M_{2}\right)\right|>|E(M)|$, a contradiction. Next we suppose that $M_{1}$ contains a single parallel pair. Assume that $M^{\prime} \in \mathcal{E} \mathcal{X}\left(M\left(K_{3,3}\right)\right)$ is a simple rank- $r_{1}$ matroid and that $\left|E\left(M^{\prime}\right)\right|>\left|E\left(\operatorname{si}\left(M_{1}\right)\right)\right|$. We can assume that the 2-sum of $M^{\prime}$ and $M_{2}$ along the basepoint $p$ is defined. Let $M^{\prime \prime}$ be the matroid obtained from $M^{\prime}$ by adding $p^{\prime}$ in parallel to $p$. From our earlier discussion we see that $p$ is not in a parallel pair in $M_{2}$. It follows that $M^{\prime \prime} \oplus_{2} M_{2}$ is simple. Moreover $M^{\prime \prime} \oplus_{2} M_{2}$ has no $M\left(K_{3,3}\right)$-minor. Furthermore $M^{\prime \prime} \oplus_{2} M_{2}$ contains strictly more elements than $M$. In either case we have a contradiction, so $\operatorname{si}\left(M_{1}\right)$ is a maximum-sized member of $\mathcal{E} \mathcal{X}\left(M\left(K_{3,3}\right)\right)$. By the same argument, so is $\operatorname{si}\left(M_{2}\right)$.

The inductive hypothesis implies that $\operatorname{si}\left(M_{i}\right) \in \mathcal{P}_{r_{i}}$ for $i=1,2$. We have already noted that $p$ is not in a parallel pair in both $M_{1}$ and $M_{2}$. From this we can deduce that $|E(M)| \leq\left|E\left(\operatorname{si}\left(M_{1}\right)\right)\right|+\left|E\left(\operatorname{si}\left(M_{2}\right)\right)\right|-1$. As $M$ must be at least as large as a member of $\mathcal{P}_{r}$ we deduce that

$$
14 r / 3-\alpha(r) \leq\left|E\left(\operatorname{si}\left(M_{1}\right)\right)\right|+\left|E\left(\operatorname{si}\left(M_{2}\right)\right)\right|-1 .
$$

However, by our inductive assumption we see that $\left|E\left(\operatorname{si}\left(M_{i}\right)\right)\right| \leq 14 r_{i} / 3-$ $\alpha\left(r_{i}\right)$ for $i=1,2$. Therefore

$$
\begin{aligned}
14 r / 3-\alpha(r) & \leq 14 / 3\left(r_{1}+r_{2}\right)-\left(\alpha\left(r_{1}\right)+\alpha\left(r_{2}\right)\right)-1 \\
& =14 / 3(r+1)-\left(\alpha\left(r_{1}\right)+\alpha\left(r_{2}\right)\right)-1 .
\end{aligned}
$$

This implies that $\alpha\left(r_{1}\right)+\alpha\left(r_{2}\right)-\alpha(r) \leq 11 / 3$. Combining this with the fact that $r_{1}+r_{2}=r+1$, and examining the cases, we see that either $r_{1}$ or $r_{2}$ must be congruent to 1 modulo 3 . Without loss of generality we will assume that $r_{1} \equiv 1(\bmod 3)$. Since $\operatorname{si}\left(M_{1}\right) \in \mathcal{P}_{r_{1}}$ this means that $\operatorname{si}\left(M_{1}\right)$ is obtained by taking the parallel connection of a collection of isomorphic copies of $\mathrm{PG}(3,2)$. It follows easily from Proposition 2.4 that the parallel connection is an associative operation, and $M$ is the parallel connection of $\operatorname{si}\left(M_{1}\right)$ and a matroid from $\mathcal{P}_{r_{2}}$. Therefore $M$ belongs to $\mathcal{P}_{r}$, a contradiction.

To prove the other direction of the theorem we note that if $M \in \mathcal{P}_{r}$ for some $r \geq 2$ then $M$ is simple and $M \in \mathcal{E X}\left(M\left(K_{3,3}\right)\right)$ by Proposition 5.2. By the first part of the proof any maximum-sized rank- $r$ member 
of $\mathcal{E} \mathcal{X}\left(M\left(K_{3,3}\right)\right)$ has the same size as $M$, and therefore $M$ itself is maximumsized. This completes the proof.

\section{Critical exponents}

Suppose that $M$ is a loopless $\mathrm{GF}(q)$-representable matroid with rank $r$. Then $M$ can be considered as a multiset of points in the projective space $\mathrm{PG}(r-1, q)$. The critical exponent of $M$ over $q$ is the smallest integer $k$ such that there is a set of hyperplanes $H_{1}, \ldots, H_{k}$ in $\mathrm{PG}(r-1, q)$ with the property that $H_{1} \cap \cdots \cap H_{k}$ contains no points of $E(M)$. Finding $c(M ; q)$ is known as the critical problem (see Kung [11] for an exposition). It is easy to see that if $e$ is an element of the matroid $M$, then $c(M \backslash e ; q) \leq c(M ; q)$, for all possible $q$.

Kung [10] looked at the critical exponent over $\mathrm{GF}(2)$ of binary matroids with no $M\left(K_{3,3}\right)$-minor. He showed that if $M \in \mathcal{E X}\left(M\left(K_{3,3}\right)\right)$ is simple then $c(M ; 2) \leq 10$. In this section we show that in fact $c(M ; 2) \leq 4$, and that this bound can not be improved. In particular, we show that if $M \in \mathcal{E} \mathcal{X}\left(M\left(K_{3,3}\right)\right)$ is loopless and $c(M ; 2)=4$, then $M$ has a 3 -connected component isomorphic to $\mathrm{PG}(3,2)$.

The critical exponent can be expressed in terms of the characteristic polynomial. Suppose that $M$ is a matroid on the ground set $E$. Then the characteristic polynomial of $M$, denoted $\chi(M ; t)$, is defined by

$$
\chi(M ; t)=\sum_{A \subseteq E}(-1)^{|A|} t^{r(M)-r(A)} .
$$

Suppose that $M$ is a loopless $\mathrm{GF}(q)$-representable matroid. Then $\chi\left(M ; q^{k}\right) \geq 0$ for every positive integer $k$, and $c(M ; q)$ is the least positive integer $k$ such that $\chi\left(M ; q^{k}\right)$ is positive. It is known that if $G$ is a graph, then the flow polynomial of $G$, denoted $F(G ; t)$, is equal to $\chi\left(M^{*}(G) ; t\right)$.

Lemma 6.1. Suppose that $M$ is an internally 4-connected member of $\mathcal{E X}\left(M\left(K_{3,3}\right)\right)$. Then $c(M ; 2) \leq 4$, and if $c(M ; 2)=4$, then $M$ is isomorphic to $\mathrm{PG}(3,2)$.

Proof. It is easy to see that $\mathrm{PG}(3,2)$ has critical exponent 4 over $\mathrm{GF}(2)$ (see [11, Section 8.1]).

Assume that $M$ is an internally 4-connected member of $\mathcal{E} \mathcal{X}\left(M\left(K_{3,3}\right)\right)$ other than $\mathrm{PG}(3,2)$. Suppose that $M=M^{*}(G)$ is a cographic matroid. Jaeger's 8-flow theorem [8] shows that $\chi(M ; 8)=F(G ; 8)>0$, and hence $c(M ; 2) \leq 3$. Now suppose that $M \cong \Delta_{r}$, a triangular Möbius matroid. Consider the GF(2)-representation of $\Delta_{r}$ discussed in Section 2.5. Suppose that each point of $\mathrm{PG}(r-1,2)$ corresponds to a vector $\left(x_{1}, \ldots, x_{r}\right)$. Let $H_{1}, H_{2}$, and $H_{3}$ be hyperplanes of PG $(r-1,2)$ defined, respectively, by the equations $x_{r}=0, x_{1}+\cdots+x_{r}=0$, and either $x_{1}+x_{3}+\cdots+x_{r-1}=0$ or $x_{1}+x_{3}+\cdots+x_{r-2}=0$, depending on whether $r$ is even or odd. It is easy to see that no point of $\Delta_{r}$ is contained in all of $H_{1}, H_{2}$, and $H_{3}$, so $c(M ; 2) \leq 3$. 
Suppose that $M \cong \Upsilon_{r}$, a triadic Möbius matroid. Consider the representation of $\Upsilon_{r}$ in Section 2.5. No point of $\Upsilon_{r}$ is contained in the hyperplane defined by $x_{1}+\cdots+x_{r}=0$, so $c(M ; 2) \leq 1$.

Finally we suppose that $M$ is isomorphic to one of the sporadic matroids in Theorem 1.1. The largest such matroid with rank 4 is $\operatorname{PG}(3,2)$, and it known that every proper minor of this matroid has critical exponent at most three over GF(2) [11, Section 8.1]. Thus we will suppose that $r(M) \geq 5$. The sporadic matroid $T_{12}$ has rank 6 . By examining the matrix representation of $T_{12}$ in [12, Appendix B], we see that no point of $T_{12}$ is contained in the hyperplane defined by $x_{1}+\cdots+x_{6}=0$. Thus $c\left(T_{12} ; 2\right) \leq 1$. Let $A$ be the matrix in $[12$, Appendix $\mathrm{B}]$ such that $\left[I_{5} \mid A\right]$ represents the rank-5 sporadic matroid $M_{5,12}^{a}$. If

$$
H_{5,12}^{a}=\left[\begin{array}{ccccc}
1 & 1 & 0 & 0 & 0 \\
1 & 0 & 1 & 0 & 1 \\
1 & 1 & 1 & 1 & 1
\end{array}\right]
$$

then $H_{5,12}^{a}\left[I_{5} \mid A\right]$ contains no zero columns. This means that no point of $M_{5,12}^{a}$ is contained in all three of the hyperplanes defined by $x_{1}+x_{2}=0$, $x_{1}+x_{3}+x_{5}=0$, and $x_{1}+x_{2}+x_{3}+x_{4}+x_{5}=0$. Thus $c\left(M_{5,12}^{a} ; 2\right) \leq 3$.

In the same way we can show that $M_{5,13}, M_{6,13}, M_{7,15}, M_{9,18}$, and $M_{11,21}$ all have critical exponent at most three by examining the matrices

$$
\begin{gathered}
H_{5,13}=\left[\begin{array}{lllll}
1 & 0 & 0 & 0 & 1 \\
1 & 0 & 1 & 0 & 1 \\
1 & 1 & 1 & 1 & 0
\end{array}\right] H_{6,13}=\left[\begin{array}{cccccc}
0 & 1 & 0 & 0 & 0 & 0 \\
1 & 0 & 0 & 1 & 1 & 0 \\
1 & 1 & 1 & 1 & 1 & 1
\end{array}\right] \\
H_{7,15}=\left[\begin{array}{lllllll}
1 & 0 & 1 & 0 & 1 & 0 & 1 \\
1 & 1 & 1 & 1 & 1 & 1 & 1
\end{array}\right] H_{9,18}=\left[\begin{array}{lllllllll}
1 & 1 & 0 & 0 & 0 & 0 & 0 & 0 & 0 \\
1 & 0 & 1 & 0 & 1 & 0 & 1 & 0 & 1 \\
1 & 1 & 1 & 1 & 1 & 1 & 1 & 1 & 1
\end{array}\right]
\end{gathered}
$$

and

$$
H_{11,21}=\left[\begin{array}{lllllllllll}
0 & 0 & 0 & 0 & 0 & 0 & 1 & 0 & 0 & 1 & 0 \\
1 & 0 & 1 & 0 & 1 & 0 & 0 & 0 & 1 & 1 & 1 \\
1 & 1 & 1 & 1 & 1 & 1 & 1 & 1 & 1 & 1 & 1
\end{array}\right]
$$

As every sporadic matroid in Theorem 1.1 can be produced from one of $\mathrm{PG}(3,2), M_{5,12}^{a}, M_{5,13}, T_{12}, M_{6,13}, M_{7,15}, M_{9,18}$, or $M_{11,21}$ by deleting elements, the proof is complete.

Next we come to the main result of this section.

Theorem 6.2. Suppose that $M \in \mathcal{E} \mathcal{X}\left(M\left(K_{3,3}\right)\right)$ is a loopless matroid. Then $c(M ; 2) \leq 4$, and if $c(M ; 2)=4$, then either

(i) $M$ is isomorphic to $\mathrm{PG}(3,2)$; or,

(ii) $M$ can be expressed as the 1- or 2-sum of $M_{1}$ and $M_{2}$, where $M_{1}, M_{2}$ belong to $\mathcal{E X}\left(M\left(K_{3,3}\right)\right)$, and either $c\left(M_{1} ; 2\right)=4$ or $c\left(M_{2} ; 2\right)=4$.

Proof. Suppose that $M$ is a minor-minimal counterexample to the theorem. Lemma 6.1 shows that $M$ cannot be internally 4-connected. Assume that $M$ is not connected, so that $M$ can be expressed as $M_{1} \oplus M_{2}$. Clearly $M_{1}$ 
and $M_{2}$ are loopless members of $\mathcal{E} \mathcal{X}\left(M\left(K_{3,3}\right)\right)$. It is well known, and easy to verify, that $\chi(M ; t)=\chi\left(M_{1} ; t\right) \chi\left(M_{2} ; t\right)$. If $c(M ; 2)>4$ then $\chi(M ; 16)=0$, implying that $\chi\left(M_{i} ; 16\right)=0$ for some $i \in\{1,2\}$. But $M_{i}$ is a proper minor of $M$, so we have a contradiction to minimality. Therefore we suppose that $c(M ; 2)=4$, so that $\chi(M ; 8)=0$. This implies that $c\left(M_{i} ; 2\right)=4$ for some $i \in\{1,2\}$, and $M$ satisfies statement (ii).

Now we must assume that $M$ is connected. Suppose that $M$ can be expressed as the 2-sum of $M_{1}$ and $M_{2}$ along the basepoint $p$, by Proposition 2.11. Again $M_{1}$ and $M_{2}$ are loopless members of $\mathcal{E} \mathcal{X}\left(M\left(K_{3,3}\right)\right)$. Walton and Welsh $[24,(7)]$ note that the following relation holds:

$$
\chi(M ; t)=\frac{\chi\left(M_{1} ; t\right) \chi\left(M_{2} ; t\right)}{t-1}+\chi\left(M_{1} / p ; t\right) \chi\left(M_{2} / p ; t\right) .
$$

Since $M_{1}$ and $M_{2}$ are isomorphic to minors of $M$ it follows that $\chi\left(M_{i} ; 16\right)>$ 0 for $i=1,2$. Now $(6)$ implies that $\chi(M ; 16)>0$, so $c(M ; 2) \leq 4$. Therefore it must be the case that $c(M ; 2)=4$, so that $\chi(M ; 8)=0$. Since $\chi\left(M_{1} ; 8\right) \geq$ 0 and $\chi\left(M_{2} ; 8\right) \geq 0$ it follows that either $\chi\left(M_{1} ; 8\right)=0$ or $\chi\left(M_{2} ; 8\right)=0$. Then $M$ satisfies statement (ii) of the theorem, so we have a contradiction.

Now we must assume that $M$ is 3-connected, so $M=M_{1} \oplus_{3} M_{2}$ for some matroids $M_{1}$ and $M_{2}$. Proposition 2.13 implies that $M_{1}$ and $M_{2}$ are isomorphic to proper minors of $M$. Moreover $M_{1}$ and $M_{2}$ are loopless, and both $\operatorname{si}\left(M_{1}\right)$ and $\operatorname{si}\left(M_{2}\right)$ are 3-connected by Proposition 2.14 .

Suppose that the 3 -sum of $M_{1}$ and $M_{2}$ is along the triangle $T$, where $T=\{a, b, c\}$. The following equality is from Walton and Welsh [24].

$$
\begin{aligned}
\chi(M ; t)=\frac{\chi\left(M_{1} ; t\right) \chi\left(M_{2} ; t\right)}{(t-1)(t-2)}+\chi(M \backslash a \backslash b / c ; t) & \\
& +\chi(M \backslash a / b ; t)+\chi(M / a ; t) .
\end{aligned}
$$

All the matroids $M_{1}, M_{2}, M \backslash a \backslash b / c, M \backslash a / b$, and $M / a$ have critical exponent at most four, so the characteristic polynomial of each of these matroids, evaluated at 16 , produces a positive answer. Therefore $\chi(M ; 16)>0$ and $c(M ; 2) \leq 4$.

It must be the case that $c(M ; 2)=4$, so that $\chi(M ; 8)=0$. Then the terms of the sum in (7) must be zero at the point $t=8$. In particular, we can assume by relabeling that $\chi\left(M_{1} ; 8\right)=0$ and $c\left(M_{1} ; 2\right)=4$.

The critical exponent of $\operatorname{si}\left(M_{1}\right)$ is precisely the critical exponent of $M_{1}$. Since $\operatorname{si}\left(M_{1}\right)$ is 3-connected and obeys the theorem, it follows that $\operatorname{si}\left(M_{1}\right)$ is isomorphic to $\mathrm{PG}(3,2)$. Now $\mathrm{PG}(3,2)$ has no allowable triangles (see [12, Appendix C]). Therefore $\Delta_{T}\left(\operatorname{si}\left(M_{1}\right)\right)$ has an $M\left(K_{3,3}\right)$-minor, so $\Delta_{T}\left(M_{1}\right)$ has an $M\left(K_{3,3}\right)$-minor. Proposition 2.15 implies that $\Delta_{T}\left(M_{1}\right)$ is isomorphic to a minor of $M$, so $M$ has an $M\left(K_{3,3}\right)$-minor. This contradiction completes the proof. 


\section{REFERENCES}

[1] R. E. Bixby and W. H. Cunningham. Matroid optimization and algorithms. In Handbook of combinatorics, Vol. 1, 2, pp. 551-609. Elsevier, Amsterdam (1995).

[2] T. Brylawski. Modular constructions for combinatorial geometries. Trans. Amer. Math. Soc. 203 (1975), 1-44.

[3] B. Courcelle. On context-free sets of graphs and their monadic second-order theory. In Graph-grammars and their application to computer science (Warrenton, VA, 1986), volume 291 of Lecture Notes in Comput. Sci., pp. 133-146. Springer, Berlin (1987).

[4] J. Edmonds. Submodular functions, matroids, and certain polyhedra. In Combinatorial Structures and their Applications (Proc. Calgary Internat. Conf., Calgary, Alta., 1969), pp. 69-87. Gordon and Breach, New York (1970).

[5] J. Geelen, B. Gerards, and G. Whittle. Towards a matroid-minor structure theory. In Combinatorics, complexity, and chance, volume 34 of Oxford Lecture Ser. Math. Appl., pp. 72-82. Oxford Univ. Press, Oxford (2007).

[6] D. W. Hall. A note on primitive skew curves. Bull. Amer. Math. Soc. 49 (1943), 935-936.

[7] P. Hliněný. Branch-width, parse trees, and monadic second-order logic for matroids. J. Combin. Theory Ser. B 96 (2006), no. 3, 325-351.

[8] F. Jaeger. On nowhere-zero flows in multigraphs. In Proceedings of the Fifth British Combinatorial Conference (Univ. Aberdeen, Aberdeen, 1975). Utilitas Math., Winnipeg, Man. (1976) pp. 373-378. Congressus Numerantium, No. XV.

[9] K.-i. Kawarabayashi and B. Mohar. Some recent progress and applications in graph minor theory. Graphs Combin. 23 (2007), no. 1, 1-46.

[10] J. P. S. Kung. Excluding the cycle geometries of the Kuratowski graphs from binary geometries. Proc. London Math. Soc. (3) 55 (1987), no. 2, 209-242.

[11] J. P. S. Kung. Critical problems. In Matroid theory (Seattle, WA, 1995), volume 197 of Contemp. Math., pp. 1-127. Amer. Math. Soc., Providence, RI (1996).

[12] D. Mayhew, G. Royle, and G. Whittle. The internally 4-connected binary matroids with no $M\left(K_{3,3}\right)$-minor. To appear in the Memoirs of the American Mathematical Society.

[13] J. Oxley, C. Semple, and D. Vertigan. Generalized $\Delta-Y$ exchange and $k$-regular matroids. J. Combin. Theory Ser. B $\mathbf{7 9}$ (2000), no. 1, 1-65.

[14] J. G. Oxley. On nonbinary 3-connected matroids. Trans. Amer. Math. Soc. 300 (1987), no. 2, 663-679.

[15] J. G. Oxley. Matroid theory. Oxford University Press, New York (1992).

[16] H. Qin and X. Zhou. The class of binary matroids with no $M\left(K_{3,3}\right)^{-}, M^{*}\left(K_{3,3}\right)$-, $M\left(K_{5}\right)$ - or $M^{*}\left(K_{5}\right)$-minor. J. Combin. Theory Ser. B 90 (2004), no. 1, 173-184.

[17] N. Robertson and P. D. Seymour. Graph minors. XIII. The disjoint paths problem. J. Combin. Theory Ser. B 63 (1995), no. 1, 65-110.

[18] P. D. Seymour. Decomposition of regular matroids. J. Combin. Theory Ser. B 28 (1980), no. 3, 305-359.

[19] P. D. Seymour. Recognizing graphic matroids. Combinatorica 1 (1981), no. 1, 75-78.

[20] K. Truemper. On the efficiency of representability tests for matroids. European J. Combin. 3 (1982), no. 3, 275-291.

[21] K. Truemper. A decomposition theory for matroids. V. Testing of matrix total unimodularity. J. Combin. Theory Ser. B 49 (1990), no. 2, 241-281.

[22] W. T. Tutte. An algorithm for determining whether a given binary matroid is graphic. Proc. Amer. Math. Soc. 11 (1960), 905-917.

[23] K. Wagner. Über eine Eigenschaft der ebenen Komplexe. Math. Ann. 114 (1937), no. $1,570-590$.

[24] P. N. Walton and D. J. A. Welsh. On the chromatic number of binary matroids. Mathematika 27 (1980), no. 1, 1-9. 